OPEN ACCESS

Edited by:

Giorgia Mori

The University of Queensland,

Australia

Reviewed by:

Shibali Das,

Washington University School of Medicine in St. Louis, United States

Wonsik Lee,

Sungkyunkwan University,

South Korea

*Correspondence:

Jarosław Dziadek

jdziadek@cbm.pan.pl

Alina Minias

aminias@cbm.pan.pl;

alinagorna@gmail.com

Specialty section:

This article was submitted to Antimicrobials, Resistance and

Chemotherapy,

a section of the journal

Frontiers in Microbiology

Received: 16 October 2020 Accepted: 30 December 2020 Published: 02 February 2021

Citation:

Minias A, Żukowska L, Lechowicz E,

Gasior F, Knast A, Podlewska S,

Zygała D and Dziadek J (2021) Early

Drug Development and Evaluation of

Putative Antitubercular Compounds

in the -Omics Era.

Front. Microbiol. 11:618168.

doi: 10.3389/fmicb.2020.618168

\section{Early Drug Development and Evaluation of Putative Antitubercular Compounds in the -Omics Era}

\author{
Alina Minias ${ }^{1 *}$, Lidia Żukowska ${ }^{1,2}$, Ewelina Lechowicz ${ }^{1,3}$, Filip Gasior ${ }^{1,2}$, Agnieszka Knast $^{1,4}$, \\ Sabina Podlewska ${ }^{5,6}$, Daria Zygała ${ }^{1,3}$ and Jaroslaw Dziadek ${ }^{1 *}$ \\ 'Laboratory of Genetics and Physiology of Mycobacterium, Institute of Medical Biology, Polish Academy of Sciences, Lodz, \\ Poland, ${ }^{2}$ BioMedChem Doctoral School of the University of Lodz and the Institutes of the Polish Academy of Sciences in \\ Lodz, Lodz, Poland, ${ }^{3}$ Institute of Microbiology, Biotechnology and Immunology, Faculty of Biology and Environmental \\ Protection, University of Lodz, Lodz, Poland, ${ }^{4}$ Institute of Molecular and Industrial Biotechnology, Faculty of Biotechnology \\ and Food Sciences, Lodz University of Technology, Lodz, Poland, ${ }^{5}$ Department of Technology and Biotechnology of Drugs, \\ Jagiellonian University Medical College, Krakow, Poland, ${ }^{6}$ Maj Institute of Pharmacology, Polish Academy of Sciences, \\ Krakow, Poland
}

Tuberculosis (TB) is an infectious disease caused by the bacterium Mycobacterium tuberculosis. According to the WHO, the disease is one of the top 10 causes of death of people worldwide. Mycobacterium tuberculosis is an intracellular pathogen with an unusually thick, waxy cell wall and a complex life cycle. These factors, combined with M. tuberculosis ability to enter prolonged periods of latency, make the bacterium very difficult to eradicate. The standard treatment of TB requires 6-20months, depending on the drug susceptibility of the infecting strain. The need to take cocktails of antibiotics to treat tuberculosis effectively and the emergence of drug-resistant strains prompts the need to search for new antitubercular compounds. This review provides a perspective on how modern -omic technologies facilitate the drug discovery process for tuberculosis treatment. We discuss how methods of DNA and RNA sequencing, proteomics, and genetic manipulation of organisms increase our understanding of mechanisms of action of antibiotics and allow the evaluation of drugs. We explore the utility of mathematical modeling and modern computational analysis for the drug discovery process. Finally, we summarize how -omic technologies contribute to our understanding of the emergence of drug resistance.

Keywords: Mycobacterium, tuberculosis, proteomics, DNA sequencing, transcriptomics, mutagenesis, drug evaluation, drug identification pipeline

\section{INTRODUCTION}

Tuberculosis (TB) is an infectious disease caused by Mycobacterium tuberculosis. The disease is one of the top 10 causes of death of people, according to the WHO. Each year, about 10 million people fall ill with TB, and 1.5 million people die. WHO estimates that approximately a quarter of the world population is infected with $M$. tuberculosis, and $5-10 \%$ of people will develop active TB during their lifetime. Incidence rates are reported in all countries and age groups. The disease affects mostly men (57\%). Women account for $32 \%$ of cases. 
About $11 \%$ of cases are children under 15 years of age. People with weakened immune systems are at higher risk of developing the disease, with particular emphasis on people infected with HIV. They are about 19 times more prone to TB. Further factors influencing TB's risk are malnutrition, diabetes, and smoking (World Health Organization, 2019).

Mycobacterium tuberculosis is an intracellular pathogen with an unusually thick, waxy cell wall and a complex life cycle. The bacteria are transmitted by aerosol droplets and most often infect the lungs. Generally, M. tuberculosis infects alveolar macrophages, but it can also infect other respiratory system cells. The disease may also be extrapulmonary. Mycobacterium tuberculosis can infect cells of bones, genitourinary tract, skin, joints, and meninges (Lee, 2015). Mycobacteria invade macrophages and settle the infection by blocking the maturation of phagosomes. The infection of macrophages results in the host response, where various types of immune cells infiltrate the infection site. The influx of immune cells may result in the eradication of the bacteria. Incomplete eradication of bacteria progresses the disease to the latent stage. Mycobacterium tuberculosis becomes enclosed in compact and sometimes calcified cell aggregates. Mycobacterium tuberculosis slows down its metabolism due to the restriction of the influx of nutrients and oxygen. The disease becomes latent. Mycobacterium tuberculosis can persist in the infected individual's lungs for decades. When the immunity of the person wanes, granulomas liquefy, and bacteria reactivate to the active phase of the disease. The complex life cycle, intracellular life niche, thick cell wall, and the ability to enter prolonged periods of latency make M. tuberculosis very difficult to eradicate.

\section{Current Antitubercular Chemotherapy}

The standard treatment of tuberculosis requires 6-20months, depending on the drug susceptibility of the infecting strain. Antibiotics must be taken in combination, as administering a single antibiotic quickly results in pathogen drug resistance. There are four first-line drugs against tuberculosis (isoniazid - INH, rifampicin - RMP, ethambutol - EMB, pyrazinamide - PZA) and nearly 20 s-line drugs, which can be administered during the treatment of drug-resistant tuberculosis. The numbers of multidrugresistant (MDR-TB) and extensively drug-resistant (XDR-TB) strains of $M$. tuberculosis are a major problem for current antitubercular therapy. MDR-TB is resistant to two first-line drugs, RMP and IHN. XDR-TB is resistant to four core antitubercular drugs, followed by resistance to capreofluoroquinolones and one of the three injectable second-line drugs, e.g., amikacin, capreomycin (CM), or kanamycin (KAN). Nearly 484,000 cases of MDR cases of tuberculosis are estimated to exist worldwide. A total of 13,068 cases of XDR-TB were reported by 81 countries, of which most of them were from the WHO European Region and the SouthEast Asia Region. Detection of MDR-TB first requires confirmation of TB, followed by testing for drug resistance. In 2018, 51\% of people with bacteriologically confirmed TB were tested for RMP resistance (up from 41\% in 2017). Even though between 2017 and 2018, there was progress in testing, detection, and treatment of MDR-TB, only $56 \%$ of MDR cases were successfully treated globally and only $39 \%$ of cases of XDR-TB. As of 2020, WHO recommends that MDR-TB patients are to be treated with fully oral drug regimens. Injectable agents should only be used if other options are not possible. Two such agents, KAN and CM, are no longer recommended (World Health Organization, 2019). High numbers of TB patients, including patients infected with drugresistant $M$. tuberculosis justify the need to search new antitubercular compounds that could be introduced to antitubercular chemotherapy.

\section{Prospective Antitubercular Chemotherapy}

For a long time, the development of XDR-TB left patients without further options for treatment. The principal drugs for the treatment of tuberculosis were discovered between the 1940s and 1970s (streptomycin, para-aminosalicylic acid, INH, cycloserine - CS, KAN, RMP, and others; Murray et al., 2015). The path of drug discovery is long and costly (Figure 1). The process starts with the early drug discovery stage. Here, researchers identify potential inhibitors in laboratory conditions and assess their principal biological impact. The next stage is preclinical studies. This time chemicals are tested not only on bacteria but also on cell lines or live animals. The knowledge gained in this phase is helpful in Phase III of the study, which considers the drug doses tested here in later human studies. Several experimental tools are used in the preclinical stage. One is the in vitro hollow-fiber system that provides data to improve animal experimentation. The great advantage is the integration of this data with data from many different animal models. Such models are the well-known BALB/c mice and the newer Kramnik mouse model, or the marmoset and rabbit models. The preclinical stage provides valuable information about the activity of the tested drug or sterilization of the pathogen. Unfortunately, it provides limited information on the pharmacokinetics and pharmacodynamics of the drug (Dooley et al., 2019).

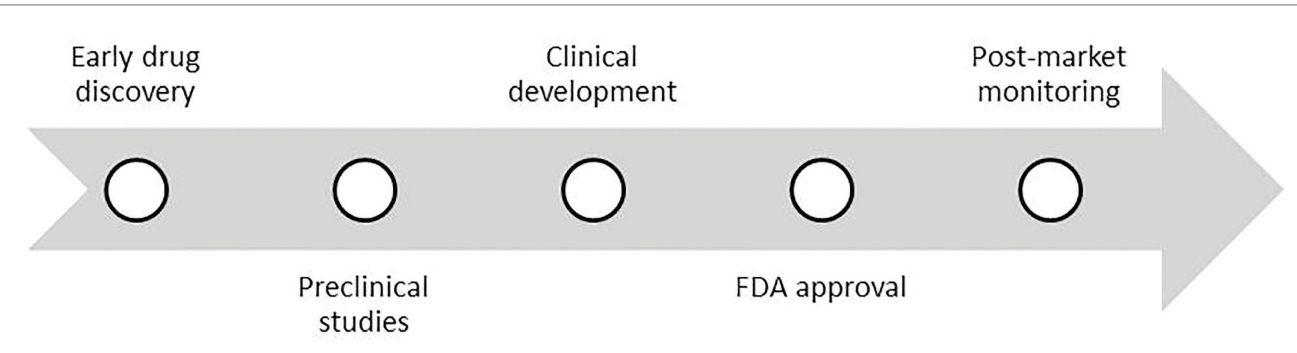

FIGURE 1 | The schematic overview of the drug discovery process. 
The main cause of failures in the clinical development of drugs is its insufficient effectiveness, which is associated with an accurate determination of pharmacokinetics (Muliaditan et al., 2017). Before entering clinical trials, it is essential to gather much information about the exposure-response relationship and the relationship between pharmacokinetics and toxicity of the tested substance. Phase I clinical trials provide information on pharmacokinetics and safety, an important element here is to establish drug interactions with food intake or whether the dose depends on the patient's body weight. Phase II studies provide detailed inter-population information. In Phase III, drug efficacy is assessed by collecting post-drug exposure data, timed microbial response, and safety (Dooley et al., 2019). After clinical trials, the development of a new drug requires approval by the Food and Drug Administration (FDA).

In 2012, the United States FDA approved the use of a novel antitubercular drug bedaquiline - BDQ. BDQ was discovered by the pharmaceutical company Johnson and Johnson, under the brand name is Sirturo (Deoghare, 2013). It is the first member of a new class of drugs called diarylquinolines. BDQ is a bactericidal drug. BDQ blocks proton pump of ATP synthase, encoded by gene atpE. ATP production is essential for cellular energy turnout (Koul et al., 2008; Deoghare, 2013). $\mathrm{BDQ}$ is recommended strictly for the treatment of MDR-TB, and when options to treat this condition using existing drugs have been exhausted. BDQ should not be used to treat latent TB infection. It should not be used alone but as part of combination therapy and never added alone to a failing regimen. By the end of 2018, 90 countries reported having imported or using BDQ (World Health Organization, 2019).

In 2014, European Medicine Agency (EMA) conditionally approved a second novel antitubercular medicine, delamanid (DLM), as a part of combination therapy to treat adults with MDR-TB. The brand name of DLM is Deltyba. DLM exhibits a low minimum inhibitory concentration, distinguishing itself from other clinically approved drugs. This medicine is a pro-drug that requires metabolic activation for its action. It is activated by the deazaflavin F420-dependent nitroreductase. Resistance against DLM includes mutations in genes participating in pro-drug activation or associated with the cofactor Ddn biosynthetic pathway.
DLM is known for specifically inhibiting the synthesis of two mycolic acids - keto mycolic acid and methoxy mycolic acid. They are the building components of the mycobacterial cell wall (absent in Gram-positive or Gram-negative bacteria). These components are also making it difficult for medicines to penetrate the cells. The use of DLM allows for more effective treatment through disrupting cell wall and shortening a treatment regimen (Tiberi et al., 2018b; Bahuguna and Rawat, 2020). By the end of 2018, 57 countries reported having imported or started using DLM (World Health Organization, 2019).

Approval of new medicine in Phase III clinical trials is always a risk, but benefits are perceived as more significant. BDQ was the first member of a new class of medicines. The principal study showed that treatment with Situro was effective; the drug worked well and fast. BDQ and DLM are well-working antibiotics, but there is already resistance against BDQ. In particular, they are mutations in atpE, gene coding ATP synthase subunit c, the target of $\mathrm{BDQ}$, or gene Rv0678, which plays a role in regulating the expression of the MmpS5-MmpL5 efflux pump (Nguyen et al., 2018; Ghajavand et al., 2019). New antibiotics are most effective in a few first years before resistance is developed and disseminated across the bacterial population. Therefore it is vital to search for new medicines. As of October 2020, a few antitubercular drugs are currently in phase III or II clinical trials (Table 1). In addition to new drugs, there are also repurposed drugs like clofazimine (CFM), levofloxacin (LFX), moxifloxacin (MFX), and linezolid (LZD), which are in phase II and phase III trials for TB too. Drugs are also tested for repurposing from the treatment of other diseases, auranofin that is an antirheumatic agent and nitazoxanide that is an antiprotozoal agent. The growing TB epidemic again developed an interest in CFM, which now is an important constituent of newer TB regimens. CFM is a pro-drug, but the exact mechanism of action is not yet known. MFX is investigated in regimens combining BDQ, pretomanid, and PZA, or rifapentine (Bahuguna and Rawat, 2020).

\section{Host-Directed Therapy}

Treatment of $M$. tuberculosis infection with currently available antibiotics has several negative features associated with drug toxicity,

TABLE 1 | New drugs in phase III and II clinical trials (Vjecha et al., 2018; Bahuguna and Rawat, 2020).

\begin{tabular}{|c|c|c|c|c|}
\hline Drug & Chemical class & Target & Effect & Clinical status \\
\hline Bedaquiline & Diarylquinoline & ATP synthase & $\begin{array}{l}\text { Inhibits energy metabolism of } \\
\text { the cell }\end{array}$ & Phase $\| I^{*}$ \\
\hline Delamanid & Nitroimidazole & Exact target not yet known & $\begin{array}{l}\text { Inhibits mycolic acid synthesis } \\
\text { (keto and methoxy mycolic } \\
\text { acids) and cell respiration }\end{array}$ & Phase $1 \|^{*}$ \\
\hline Pretomanid & Nitroimidazole & Exact target not yet known & $\begin{array}{l}\text { Inhibition of cell wall synthesis } \\
\text { and respiratory poisoning }\end{array}$ & Phase III \\
\hline Delpazolid & Oxazolidinone & $50 S$ subunit of the ribosome & Inhibits protein synthesis & Phase II \\
\hline Sutezolid & Oxazolidinone & $50 S$ subunit of the ribosome & Inhibits protein synthesis & Phase II \\
\hline SQ109 & Diamine & MmpL3 & Inhibits cell wall synthesis & Phase II \\
\hline Macozinone (PBTZ169) & Benzothiazinone & DprE1 & Inhibits cell wall synthesis & Phase II \\
\hline Telacebec (Q203) & Imidazopyridine & Cytochrome bc1 complex & Inhibits ATP synthesis & Phase II \\
\hline
\end{tabular}

${ }^{*}$ Recently approved drugs. 
and the problem is the increasing presence of drug-resistant M. tuberculosis strains. Another approach to supporting the treatment and prevention of tuberculosis is host-directed therapy (HDT). This strategy aims to modify host response related to the development, activity, and pathogenicity of M. tuberculosis infection. The HDT agents may have immunomodulatory properties, enhance the host's immune system or influence the host's metabolic pathways, which should aid in fighting the pathogen and protect the lung tissue (Tiberi et al., 2018a). One way is to activate autophagy, which would contribute to the increased intracellular killing of M. tuberculosis. In this case, the possibility of using rapamycin, metformin, statins, vitamin $\mathrm{D}$, phenylbutyrate, carbamazepine, or valproic acid is investigated. Rapamycin inhibits the activity of mammalian target of rapamycin (mTOR), which is an inhibitor of autophagy. However, the use of this compound in therapy is limited due to the potential for side effects and its breakdown by the liver enzyme CYP3A4, which is activated by RMP, one of the first-line drugs in the treatment of tuberculosis. In addition, an increase in replication was observed in cells co-infected with HIV and H37Rv in response to rapamycin. Another potential compound is metformin, which can increase AMP-activated protein kinase and reactive oxygen species expression. These abilities contribute to activating autophagy and reducing inflammation. Statins lower lipid levels by inhibiting the enzyme $\beta$-hydroxy $\beta$-methylglutaryl-CoA, which is involved in lipid metabolism. Statins have a positive effect on the maturation of phagosomes and autophagy processes and reduce the accumulation of lipids inside cells, e.g., in macrophages, limiting the growth of the pathogen. Vitamin D and phenylbutyrate may increase the expression of LL-37, cathelicidin. Moreover, vitamin D regulates the expression of cytokines and immune mediators. Carbamazepine and valpronic acid are responsible for the activation of mTOR-independent autophagy (Dara et al., 2019; Torfs et al., 2019; Ahmed et al., 2020).

Host-directed therapy may also target the disintegration of the granuloma structure. Etanercept, an inhibitor of tumor necrosis factor $\alpha$ (TNF- $\alpha$ ) involved in the formation and maintenance of granuloma, may help treat tuberculosis. Another possible drug is bevacizumab targeting vascular endothelial growth factor (VEGF). The drug influences the normalization of the vessels, which in turn causes a change in the morphology of the granuloma and the possibility of interaction with anti-tuberculosis drugs.

An important path of HDT is immunomodulation, increasing the anti-inflammatory response, which would help to reduce tissue damage. Ibuprofen, diclofenac, acetylsalicylic acid, and vitamin D are of interest (Torfs et al., 2019; Ahmed et al., 2020). Nonsteroidal anti-inflammatory drugs such as ibuprofen or diclofenac can reduce the inflammatory response by inhibiting cyclooxygenases. Acetylsalicylic acid activates lipoxin A4, which inhibits neutrophil migration and TNF- $\alpha$ production (Torfs et al., 2019; Ahmed et al., 2020; Young et al., 2020).

\section{Vaccination}

Bacillus Calmette-Guérin (BCG) is a vaccine based on attenuated Mycobacterium bovis, and it has been available since 1921. The BCG vaccine is currently applied worldwide, mostly in high burden countries of Africa, Asia, and South America.
In 2011, among the 180 countries with available data, 157 countries recommended universal BCG vaccination (Zwerling et al., 2011). BCG vaccine efficiency is limited, as reflected by the number of tuberculosis cases worldwide. Therefore there is an ongoing search for novel, more effective vaccines. Several types of novel vaccine candidates are currently in clinical trials. They are composed of recombinant proteins and adjuvants, they are viral vectored, and they are attenuated and whole-cell vaccines (Kaufmann, 2020). The call for new vaccines is supported by the World Health Organization. The principal recommendations are that the new vaccine should be affordable, safe, and more efficient than the current BCG vaccine in the prevention of infection, disease, or recurrence.

One of the most promising vaccine candidates is the M72 subunit vaccine developed by GlaxoSmithKline. The vaccine successfully passed a phase IIb clinical trial. It was 54\% effective (Van Der Meeren et al., 2018). The study tested booster vaccination of HIV-positive adults with latent $\mathrm{TB}$ infection who had been vaccinated with BCG as infants. This vaccine contains two TB antigens, fused in one protein and combined with AS 01E as an adjuvant. The disadvantage of this adjuvant is its high cost of production and limited availability, which may be an obstacle to the wide scale M72 vaccination (Kaufmann, 2020).

\section{Antitubercular Drug Development Market}

It takes a lot of time and cost to bring a new drug to market. The average cost that pharmaceutical companies have to bear is about US $\$ 2.6$ billion during 10 years of research and development. Clinical trials consume most of this funding, about US\$ 1-2.5 billion. Clinical trials are also the longest stage during the drug discovery process - they can last up to 6-7years. Funding for the prevention, diagnosis, and treatment of ТВ has doubled since 2006, but it is still insufficient. In 2019, 119 low- and middle-income countries funding reached US\$ 6.8 billion, up from US $\$ 3.5$ billion in 2006. Most funds (about 87\%) are available from domestic sources. Pharmaceutical companies mainly research new antibiotics. Most of them are small and medium-sized institutions (81\%). Academia carries out $12 \%$ of new antibiotic research. Large pharma companies account for 3\% of research. Non-profit institutions and public-private partnerships carry out the rest (Theuretzbacher et al., 2020). In 2019, international donor funding amounted to US\$ 0,9 billion, which is far below than what was assumed by the Stop TB Partnership's Global Plan. Most of the international donor funding comes from the Global Fund to Fight AIDS, Tuberculosis, and Malaria. According to data from Treatment Action Group, there was the funding of US\$ 772 million for TB research and development in 2017, which is much less than the target of at least US\$ 2 billion per year set at the UN high-level meeting on TB (World Health Organization, 2019). High costs born during the drug development process justify the introduction of new technological solutions, including -omic technologies, that can facilitate the introduction of new, effective, and safe drugs to the market. 


\section{STRATEGIES TO FIND NEW DRUGS FOR TUBERCULOSIS TREATMENT AND THE DRUG DISCOVERY PROCESS}

Designing a new drug and bringing it to market is a very time-consuming process that can take up many years (Hughes et al., 2011). This process is also highly costly, with little prospect of reimbursement from developing countries where tuberculosis is most prevalent. There are several important points to consider when searching for new antitubercular drugs. There is a need to provide shorter, simpler, and affordable multi-drug regimens for drug-sensitive M. tuberculosis; shorter, more effective, less toxic, and less expensive regimes for drugresistant $M$. tuberculosis; and shorter, more straightforward, easily tolerable, and safe regimes for latent tuberculosis. Furthermore, new antitubercular drugs should not antagonize other medications, such as those used during HIV infection treatment. Finally, ideal drugs are those that have restricted the occurrence of drug-resistance.

There are two major paths to discover new antibiotics during the early stage of drug development (Figure 2). The first approach involves screening libraries of chemicals to find a "hit" - a molecule that kills a pathogen at the desired concentration. The advantage of this approach is that bacterial cell growth, compound penetration, and target sensitivity are resolved at the time of identification. Once an active chemical is identified, it is vital to establish several issues to evaluate the utility of the compound properly. The first issue is the identification of the drug target protein. Pinpointing the drug target leads to an explanation of the mode of action (MOA) of the novel drug (Table 2).

Further, it facilitates the optimization of the chemical structure of the drug. Finally, identifying the drug target protein provides essential information regarding possible causes of potential drug resistance. It is also pertinent to establish if the drug generates adverse effects, like an increase in the pathogen virulence. Finally, the amount of drug resistance variants and drug resistance sources should be determined (Table 3). Following the evaluation of the influence of the drug on the bacterium, the chemical toxicity is tested against eukaryotic cells. If the chemical is toxic at low doses for the pathogen but not toxic for eukaryotic cells, the compound may be further tested for its effectiveness in intracellular infection models and animal models before reaching the clinical trial phase.

The second approach for finding new antibiotics begins with identifying a molecular target that is essential or otherwise important for the pathogen virulence. The proteins that make good targets for antibiotics are those for which mutations are often deleterious. Such an approach makes them less susceptible to the random development of resistance. Inhibitors of those

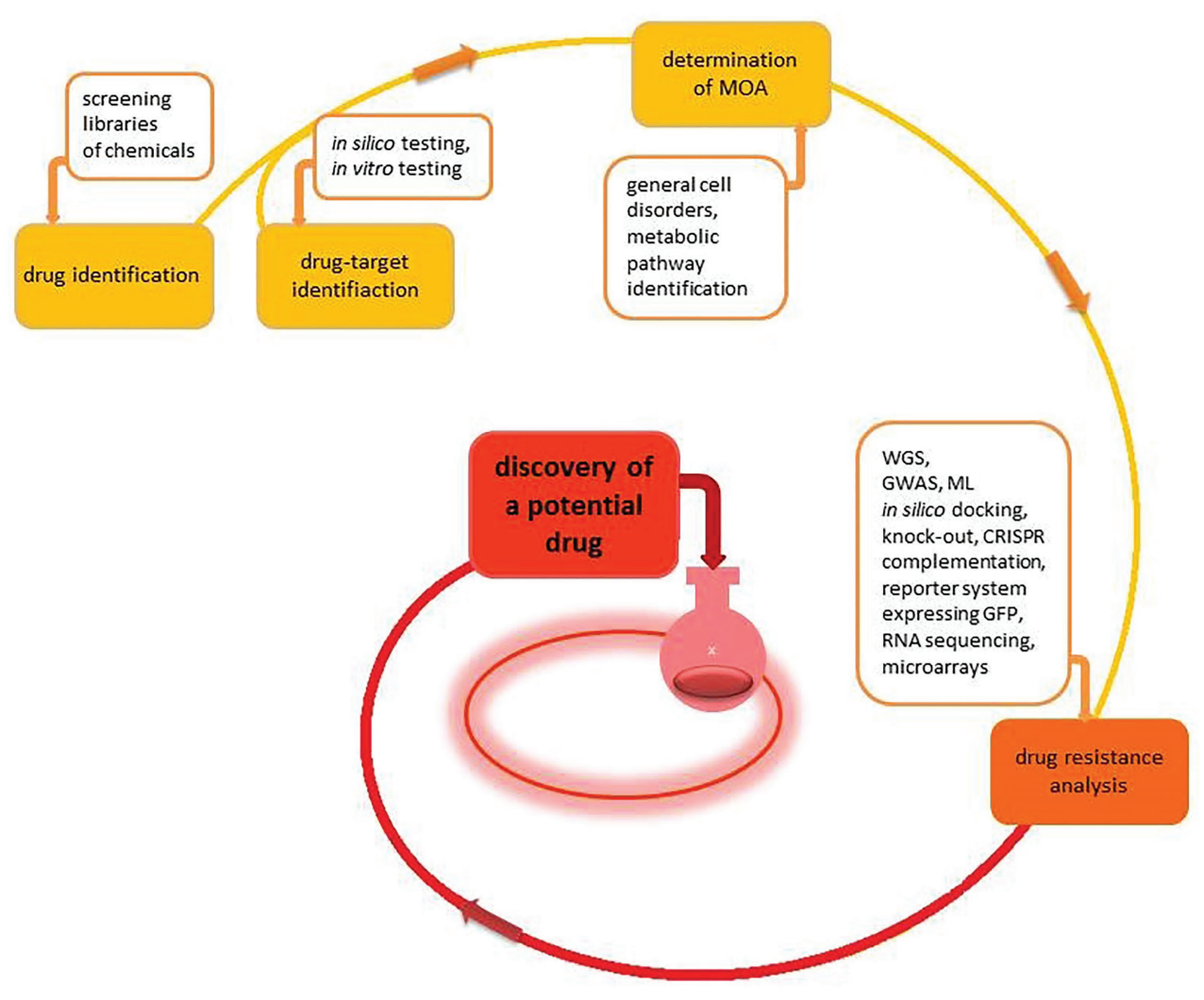

FIGURE 2 | The schematic overview of the early drug discovery process. The process begins with the identification of either a drug target or the drug itself. Next, the determination of the mode of action (MOA) and consideration of drug resistance facilitates an indication of safe and practical potential new drugs. 
TABLE 2 | Approaches to establishing MOA of new drugs.

\begin{tabular}{|c|c|c|}
\hline Aim & Methods & Example studies \\
\hline $\begin{array}{l}\text { Identification of general } \\
\text { cell disorders and } \\
\text { disrupted pathways and } \\
\text { linking them to the } \\
\text { disrupted metabolic } \\
\text { pathway } \\
\text { Identification and } \\
\text { confirmation of the drug } \\
\text { target }\end{array}$ & $\begin{array}{l}\text { RNA-Seq } \\
\text { Analysis of metabolites } \\
\text { by LC-MS } \\
\text { Analysis of lipid content } \\
\text { by LC-MS } \\
\text { WGS to identify } \\
\text { mutations in the drug } \\
\text { target } \\
\text { Generation of knock-out } \\
\text { strains } \\
\text { Generation of } \\
\text { complemented mutants } \\
\text { with increased gene } \\
\text { expression } \\
\text { Generation of } \\
\text { complemented mutants } \\
\text { with decreased gene } \\
\text { expression } \\
\text { CRISPR system gene } \\
\text { expression depletion }\end{array}$ & $\begin{array}{l}\text { Andries et al., } 2005 \\
\text { Bryk et al., } 2008 \\
\text { Kumar et al., } 2015\end{array}$ \\
\hline
\end{tabular}

TABLE 3 | Identification of sources of drug resistance.

\begin{tabular}{|c|c|c|}
\hline Aim & Methods & Example studies \\
\hline \multirow{2}{*}{$\begin{array}{l}\text { Identification of the } \\
\text { modification of the drug } \\
\text { target }\end{array}$} & $\begin{array}{l}\text { WGS of the resistant } \\
\text { strains }\end{array}$ & Takiff et al., 1994 \\
\hline & $\begin{array}{l}\text { GWAS } \\
\text { In silico docking }\end{array}$ & $\begin{array}{l}\text { Farhat et al., } 2019 \\
\text { Nachappa et al., } 2020\end{array}$ \\
\hline \multirow{4}{*}{$\begin{array}{l}\text { Identification of the } \\
\text { modification of the } \\
\text { disrupted metabolite } \\
\text { pathway }\end{array}$} & RNA-Seq & $\begin{array}{l}\text { Reviewed in Briffotaux } \\
\text { et al. (2019) }\end{array}$ \\
\hline & TraSH & Viswanathan et al., 2017 \\
\hline & PIP networks & $\begin{array}{l}\text { Raman and Chandra, } \\
2008\end{array}$ \\
\hline & GWAS and ML & $\begin{array}{l}\text { Yang et al., 2018; Deelder } \\
\text { et al., } 2019\end{array}$ \\
\hline \multirow[t]{2}{*}{$\begin{array}{l}\text { Induction of the efflux } \\
\text { pumps }\end{array}$} & $\begin{array}{l}\text { Using reporter system } \\
\text { expressing fluorescent } \\
\text { protein }\end{array}$ & Jain et al., 2016 \\
\hline & RNA-Seq & $\begin{array}{l}\text { Reviewed in Briffotaux } \\
\text { et al. (2019) }\end{array}$ \\
\hline
\end{tabular}

proteins can be found in two ways: through in silico screening using bioinformatic analyses and tested in experimental conditions (Korycka-Machala et al., 2017) or through in vitro screening inhibitor trough enzymatic/colorimetric assay (Syre et al., 2003; Grzelak et al., 2019). Potential molecular targets proposed in previous studies are, for example, proteins associated with DNA repair systems (for review, see Minias et al., 2019), DNA replication (for review, see Płocinska et al., 2017), multidrug efflux pumps (Viveiros et al., 2003; Balganesh et al., 2012), or proteins necessary for cell division (Plocinska et al., 2012; Chatterjee et al., 2018; Gorla et al., 2018).

The ongoing digital revolution introduces novel solutions to old problems. New bioinformatic technologies and the availability of these advanced technologies for research allow for significant advances in the field of drug development. This review provides a perspective on how modern -omic technologies facilitate the drug discovery process for tuberculosis treatment. We discuss how methods of DNA and RNA sequencing, proteomics, and genetic manipulation of organisms increase our understanding of mechanisms of action of antibiotics and allow the evaluation of drugs. We explore the utility of mathematical modeling and modern computational analysis for the drug discovery process. Finally, we summarize how -omic technologies contribute to our understanding of the emergence of drug resistance.

\section{IDENTIFICATION OF ANTIBACTERIAL COMPOUNDS}

\section{Screening Trough Libraries of Chemicals}

Antibacterial compounds can be identified in high-throughput screens (HTS), which involve searching through a library of chemicals against either bacterial cell culture or chosen bacterial proteins in in vitro assay. When there is an observable inhibition of growth or enzyme activity at the desired concentration, the compound is tested further. The key to every HTS endeavor is the compound collection. Libraries of compounds are designed and selected for drug-like properties and structural diversity, critical to identifying unique hits for screening targets (Lushington and Chaguturu, 2014). The main challenge in this approach is the quality of chemical libraries. When creating a library, it is important to pay attention to its composition by excluding compounds that may interfere with screening results. Libraries should not contain unstable, highly reactive, or insoluble compounds. Assessment of the identity of the relationship and validation of purity is also essential. It is important to develop the standard of libraries by engaging library creators in their development, and also scientists dealing with screening research and commercial library managers. There is a forum (Nature Chemical Biology) that brings together this community, where issues in this field are presented and standards are discussed (Screening we can believe in, 2009). Millions of compounds are now commercially available, which allows for the development of research for both academics and the pharmaceutical industry.

\section{Adjusting Culture Conditions}

Bacterial culture can be carried out in various environmental conditions. Researchers often try to adapt and resemble culture conditions to those inside the human cells during pathogen infection. Screening of compounds can be done with reporter assays using different types of culture. Grant et al. (2013) screened a library of compounds against both actively replicating and non-replicating bacilli. They constructed fluorescent reporter assays for replicating and non-replicating conditions. Screen of compounds with both assays allowed to characterize the compounds as having effect on only replicating activity, only non-replicating activity, or both replicating and non-replicating activity.

\section{Using Surrogate Models}

Working with $M$. tuberculosis brings many difficulties. For example, it is a slow-growing strain, requiring a BSL-3 (biosafety level) 
laboratory. Such aspects limit the possibilities of seeking new drug targets. A solution is the use of surrogate strain - Mycobacterium (Mycolicibacterium) smegmatis. It grows faster than M. tuberculosis, and working with it is more safe. Mycobacterium smegmatis shows good compliance with antitubercular drugs if it is grown in a low nutrient culture medium (Lelovic et al., 2020). The study by Altaf et al. (2010) involving screening of chemical libraries, showed that around $50 \%$ of inhibitors active against $M$. smegmatis are also active against $M$. tuberculosis. Another surrogate bacterium, slowgrowing $M$. bovis BCG, got much better results with the majority of correlating hits. The less common surrogate models used in the antitubercular drug development process are Mycobacterium aurum (Gupta and Bhakta, 2012) and Mycobacterium marinum (Boot et al., 2018).

\section{IDENTIFICATION OF THE DRUG TARGET AND DETERMINATION OF ITS MODE OF ACTION}

As the mechanism of action of individual antibiotics was discovered, it was understood that each of these drugs had a specific target. In addition to inhibiting their primary target, many drugs affect cell metabolism by generating toxic intermediates and triggering a cascade of molecular events, resulting in significant cell changes. Therefore, it is vital to consider the overall cellular metabolism when the cell is under the influence of the antibiotic (Kana et al., 2014).

\section{Whole-Genome Sequencing}

One of the principal paths to identify the drug target is to look for drug resistance mutations, as they often occur in the target. This can be done by whole-genome sequencing (WGS) of the resistant strain. Once the mutation is identified, it should be confirmed by the generation of drug resistant mutant after the introduction of the mutation into a drug-susceptible strain. This approach was used for target identification of BDQ. The authors generated drug-resistant variants of $M$. smegmatis and $M$. tuberculosis and sequenced their genomes. They found that the mutations in atp $\mathrm{E}$ gene are responsible for resistance to the compound. The $M$. smegmatis wild-type (WT) strain was transformed with a construct expressing the ATP synthetase subunit of the $M$. smegmatis mutant. The complementation with the mutant allele caused drug resistance (Andries et al., 2005). Recently, using a similar strategy, we identified a drug target for 1H-benzoimidazole derivatives. WGS identified mutations in the $\mathrm{mmpl} 3$ gene encoding the integral membrane protein. Strains with trans-complementation of the wild-type mutated target gene were prepared. The resulting $1 \mathrm{H}$-benzoimidazole resistance confirmed the role of the gene in the resistant phenotype (Korycka-Machała et al., 2019).

\section{Genetic Modification of Mycobacteria}

The information regarding the genomic DNA sequence provides a base for genetic manipulations. Several genetic modification approaches are currently available, including the construction of knock-out mutants, complemented mutants, and the use of reporter systems and interference systems, including the CRISPR/dCas system. Gene replacement by homologous recombination allows obtaining unmarked genetic mutants carrying large deletions within the genes of interest. These mutants can be complemented with genes of interest under native or inducible promoter (Parish and Stoker, 2000). One can also silence the gene using CRISPR/dCas (Choudhary et al., 2015). Obtaining a mutant with regulated target depletion allows performing several experiments. The use of such strain allows assessing the impact of the depletion of the studied gene in various conditions (anaerobic, acid $\mathrm{pH}$, and antibiotics). Previously, CRISPR/dCas mutants were used to analyze the MmpL3 as a therapeutic target. GoldenGate cloning was used to develop the CRISPR/dCas plJT965 plasmid. As a result, a 6-fold decrease in expression of the $m m p L 3$ gene was obtained. The mutation led to a 5-fold increase in strain sensitivity to Mmpl3 inhibitor (McNeil and Cook, 2019).

Complementation of mutants with possible drug-target genes allows control of gene expression, which can be used for the evaluation of chemical compounds against specific targets. An example of this approach is evaluating the available library of GlaxoSmith Kline compounds as CTP inhibitors of PyrG synthetase. This essential enzyme is involved in several biochemical pathways affecting several aspects of Mycobacterium physiology. Compounds were tested against M. tuberculosis conditional knockdown strain using a Pip-ON inducible system. A mutant carrying the pyrG gene under promoter induced by pristinamycin I was designed. The dependence of the action of two compounds from the analyzed library on the PyrG level confirmed that the enzyme is an intracellular target (Esposito et al., 2017). Similarly, the antitubercular activity of known compounds was confirmed by constructing conditional mutants of the tetracycline-induced pan $C$ gene, identifying the inhibitory activity of flavonoid derivatives (Abrahams et al., 2012).

To further study the drug target, it is also useful to overexpress the gene of interest. For the analysis of antifolates, an $M$. tuberculosis strain carrying the plasmid pMRN1 containing a wild copy of the $d f r A$ gene under the control of the strong promoter was constructed. A 4-fold increase in the MIC 90 strain overexpressing the gene encoding dihydrofolate reductase was observed for 17 compounds, which indicates the targeted nature of these compounds (Kumar et al., 2015). Similarly, M. smegmatis strain overexpressing MtNadD was prepared using the non-interfering plasmid pVV16 and the deletion mutant. The strains were tested in the presence of benzimidazolium (N2) derivatives. The overexpressed strain consistently showed a larger MIC compared to the mutant strain (Osterman et al., 2019).

\section{Transcriptomics}

When identifying an antibiotic's mode of action, one can look for changes in the metabolism, growth, and morphology of bacteria. These observations allow the assessment of bacterial target inhibition through comparison with known 
mechanisms (Silver, 2012). So an alternative route to identify potential drug targets is through transcriptomic data obtained either through microarrays or RNA-Seq (Fields et al., 2017). O'Rourke et al. (2020) investigated transcriptomic profiles of 37 antibiotics within six different mechanisms of action, which allowed blind predictions of the antibiotic class based on transcriptomic response with an accuracy of $<80 \%$. A similar model was developed for M. marinum (Boot et al., 2018). Betts et al. (2003) examined M. tuberculosis gene expression after treatment with $\mathrm{INH}$, triclosan, and thiolactomycin. Based on gene expression changes, a transcription profile model was proposed that enabled the determination of differences between $M$. tuberculosis treated with each of the three drugs. This model can be used to determine the MOA of uncharacterized mycolic acid biosynthesis inhibitors (Briffotaux et al., 2019). Differentially expressed genes were also evaluated in a whole blood model under the influence of RMP, INH, PZA, and EMB (Kwan et al., 2020). Currently, there are over a hundred reports of differential expression of mycobacterial genes. The results from these reports were recently combined into model INDIGO-MTB. The goal of the model was to identify antibiotic combinations that are most promising for TB drug development. The authors identified the transcription factor Rv1353c as a regulator of multiple drug interaction outcomes. They concluded that this factor could be targeted for rationally enhancing drug synergy (Ma et al., 2019).

\section{Metabolomics}

Metabolomics is a useful approach for finding new targets for antituberculosis drugs and understanding compounds' mode of action (Tuyiringire et al., 2018). By analyzing the network of metabolites and the interactions between them, it is possible to obtain knowledge about the cell processes (Goff et al., 2020). de Carvalho et al. (2010) supplemented M. tuberculosis cultures with 13C-labeled carbon substrates and then analyzed the metabolites by LC-MS, proving that Mycobacteria can catabolize multiple carbon sources simultaneously. In the following study, Prosser and Carvahlo used LC-MS to interrogate the antibiotic action mechanism of d-cycloserine (Prosser and de Carvalho, 2013). Halouska et al. (2012) described metabolomics changes in model M. smegmatis under the influence of 12 known drugs and three chemical leads. Nuclear magnetic resonance (NMR) analysis of the M. smegmatis metabolome clustered drug-induced patterns, correlating them with in vivo drug activity. Zampieri et al. (2018) analyzed the metabolomic response of $M$. smegmatis to 62 reference compounds. They used that information to predict the MOA of a library of 212 new anti-mycobacterial compounds from the pharmaceutical company GlaxoSmithKline.

A separate part of metabolomics is lipidomics, which studies the interactions between currently known lipid species and other lipids, proteins, and metabolites in the cell (Wu et al., 2014). It is based on the use of mass spectrometry (MS), a technique by which the mass-to-charge ratio and the number of ions are measured, gas chromatography (GC-MS), and liquid chromatography (LC-MS; Griffiths and Wang, 2009). Analysis of cell lipid content changes in response to changing environmental conditions may lead to the identification of key pathways in lipid biosynthesis. Pal et al. (2018) used lipidomics to show that INH treatment of M. tuberculosis can alter the composition of glycerolipids and glycerophospholipids.

\section{CHOOSING THE DRUG TARGET AND FINDING ITS INHIBITORS}

Because of a significant understanding of the processes taking place in bacterial cells, the number of potential molecular targets for inhibition is very long. Proteins taking action in DNA metabolism and cell wall synthesis are of particular interest. Other attractive targets for new drugs are proteins from the RND family (resistance, nodulation, and cell division), especially $\mathrm{Mmpl} 3$, for which analogs of EMB displayed inhibiting activity (Campaniço et al., 2018).

\section{Genetic Modification of Mycobacteria}

The alternative approach used to identify new drugs is first identifying the target and then looking for its inhibitors. It is usually done by creating knock-out mutants, complemented mutants, and/or CRISPR/dCas mutants with specific gene changes. An important aspect to bear in mind is those target proteins identified as essential may be significant for bacterial survival only under laboratory conditions and not in the infection process (Zuniga et al., 2015). Similarly, part of the mutants obtained in laboratory conditions, during growth in laboratory media, is not viable during animal infection. To test the essentiality of individual genes, the researchers infect animals with knock-out mutants. In an exemplary study, guinea pigs were inhaled with a knock-out mutant of dlaT, which product is involved in the restriction of nitric oxide-derived reactive nitrogen intermediates. The authors confirmed a vital role of dlaT in establishing infection and searched for dlaT inhibitors trough screening a library of chemicals (Bryk et al., 2008). A desirable targeting strategy for drugs is to find a target that will shorten treatment duration and reduce the incidence of tuberculosis relapses. Hu et al. analyzed the M. tuberculosis HspX protein, which they previously linked to inhibiting this organism's growth. The BALB/c mice were infected with $h s p X$ deleted mutant and the WT strain. The animals were treated with popular antibiotics. Treatment of mice infected with the $h s p X$ mutant resulted in faster clearance of bacteria from internal organs (Hu et al., 2015).

\section{Transposon Mutagenesis}

A high-throughput method of obtaining mutants is transposon site hybridization ( $\mathrm{TraSH}$ ) developed by Sassetti et al. (2003) and DeJesus et al. (2017). The knowledge about gene function comes from inserting transposons at AT sites randomly distributed across the genome into the gene and disrupting its functions. Transposon mutagenesis allowed for a search of novel molecular targets such as virulence factors, enzymes of crucial metabolic pathways, and other essential proteins (Alksne and Dunman, 2008). Transposon mutants can be used 
to simultaneously analyze a large number of mutants for survival in animal models. Transposon mutants were tested in mice, guinea pigs, and macaques (Sassetti and Rubin, 2003; Hernandez-Abanto et al., 2007; Dutta et al., 2010). In a study by Carey et al. (2018) transposon mutagenesis was used to investigate genetic requirements for the in vitro growth of clinical strains of $M$. tuberculosis and the reference M. tuberculosis strain H37Rv. The authors identified different requirements for genes in a panel of clinical strains. One of them turned out to be katG, encoding the first-line activator of INH.

Originally TraSH was performed using microarrays. Currently, a frequent variant of $\mathrm{TraSH}$ is the sequencing of the transposon insertion (Tn-seq). The method is sensitive, and it does not rely on a pre-existing array. It is important to note that transposon mutagenesis is a high-throughput method, and as such, it is affected by a certain level of inaccuracy. The analysis of constructed mutants should confirm results obtained for individual genes.

\section{Lipidomics}

In the studies conducted by Raghunandanan et al. (2019) the authors analyzed the changes in the lipid content of M. tuberculosis undergoing hypoxia and subsequent re-oxygenation. It turned out that the dormant bacteria's lipid content was drastically low and increased during oxygenation. Despite the drastic reduction in lipid synthesis pathways during hypoxia, some of them were still acting. These pathways are potential targets for antituberculosis drugs.

\section{Bioinformatic Predictions of Drug Targets}

Potential drug targets can be identified in protein-protein interaction networks (PIP) studies. Two staples of this method are the identification of the unique non-homologous proteins through the Kyoto Encyclopedia of Gene and Genome (KEGG) database or UniProt and identification of essential genes through the Database of Essential Genes (DEG; Amir et al., 2014; Melak and Gakkhar, 2014, 2015). Search of Amir et al. (2014) for unique proteins of M. tuberculosis in metabolic pathways with the KEGG database brought up five pathways consisting of 55 proteins. Selected proteins were analyzed with DEG and UniProt to choose the best candidates for new molecular targets. In another study with a similar approach done by Melak and Gakkhar, out of 1,091 essential genes, 572 were absent in the human genome. The interactome analysis with the STRING database allowed to limiting the number of possible targets. The authors then chose 131 proteins within the close neighborhood of the center of gravity of the proteome network, seeing that they function as important communicators between different metabolic pathways. Most of them were associated with cell wall metabolism. To validate this method, researchers compared their results to known and potential drug targets. Forty-three proteins were already known targets, and some were already reported as candidates (Melak and Gakkhar, 2015). One of the obstacles in this research type is that many
M. tuberculosis proteins do not have a known function or a 3D structure available. Protein structure is important to conclude the function. However, there are attempts to use proteins with unknown functions (hypothetical proteins) as molecular targets through homology modeling (Uddin et al., 2019). To solve this issue, researchers use their 3D models (Kushwaha and Shakya, 2010) or use The Protein Data Bank (PDB; Melak and Gakkhar, 2015).

\section{Bioinformatic Predictions of Target Inhibitors}

The number of chemicals that are required for screening in order to find an appropriate inhibitor can be overwhelming. Therefore HTS is often facilitated by virtual screening (VS; Figure 3). VS utilizes computational methods to screen through ligands libraries to find new hits (Kar and Roy, 2013). Molecular docking and pharmacophore modeling are the most commonly used tools (Macalino et al., 2020), being the basis for the distinction of VS approaches into the structure- and ligandbased path. When information about the arrangement of the target atoms is available (e.g., thanks to the presence of the respective crystal structures), it is usually used for docking. Otherwise, the target structure needs to be modeled using homology or de novo (restricted to small proteins) modeling (Schmidt et al., 2014). Docking enables rough estimation of compound affinity to the target and making compound comparisons based on the quality of fitting to the binding pocket. Ligand-based VS also uses a quantitative structureactivity relationship (QSAR) analysis for predicting the activity and physicochemical properties of new potential drugs (Nowosielski et al., 2013; Adeniji et al., 2018). Similarity search approaches look for compounds with similar structures to already known ligands, according to the assumption that compounds with similar chemical structures should induce similar biological effects (Martin et al., 2002).

Virtual screening is an useful tool to identify new inhibitors for the key cellular components. For example, VS identified potential inhibitors of MraY, which is necessary for peptidoglycan synthesis (Mallavarapu et al., 2019). Other important cellular components are proteins containing the most frequently occurring drug-resistance mutations like InhA, FabD, and AhpC, which are INH targets. Through the use of VS, researchers can identify new potential drug candidates and limit the number of compounds that need testing in experimental conditions (Jagadeb et al., 2019).

Alternatively, molecular docking aids in finding more effective antitubercular drugs, which structure is based on already existing compounds with proven abilities to inhibit or completely stop $M$. tuberculosis growth. An example of such a study regarding the antitubercular drugs is the search for new inhibitors of arabinosyltransferase $\mathrm{C}$ enzyme (EmbC). EmbC participates in the formation of the cell wall, and it is probably the target of EMB. Researchers performed molecular docking of five new derivatives of the EMB. Based on bioinformatic results, the authors indicated two of them that should bind to the EmbC with higher affinity (Das et al., 2020). 
A

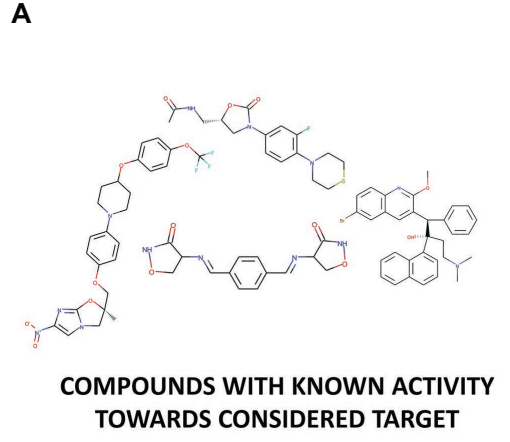

B
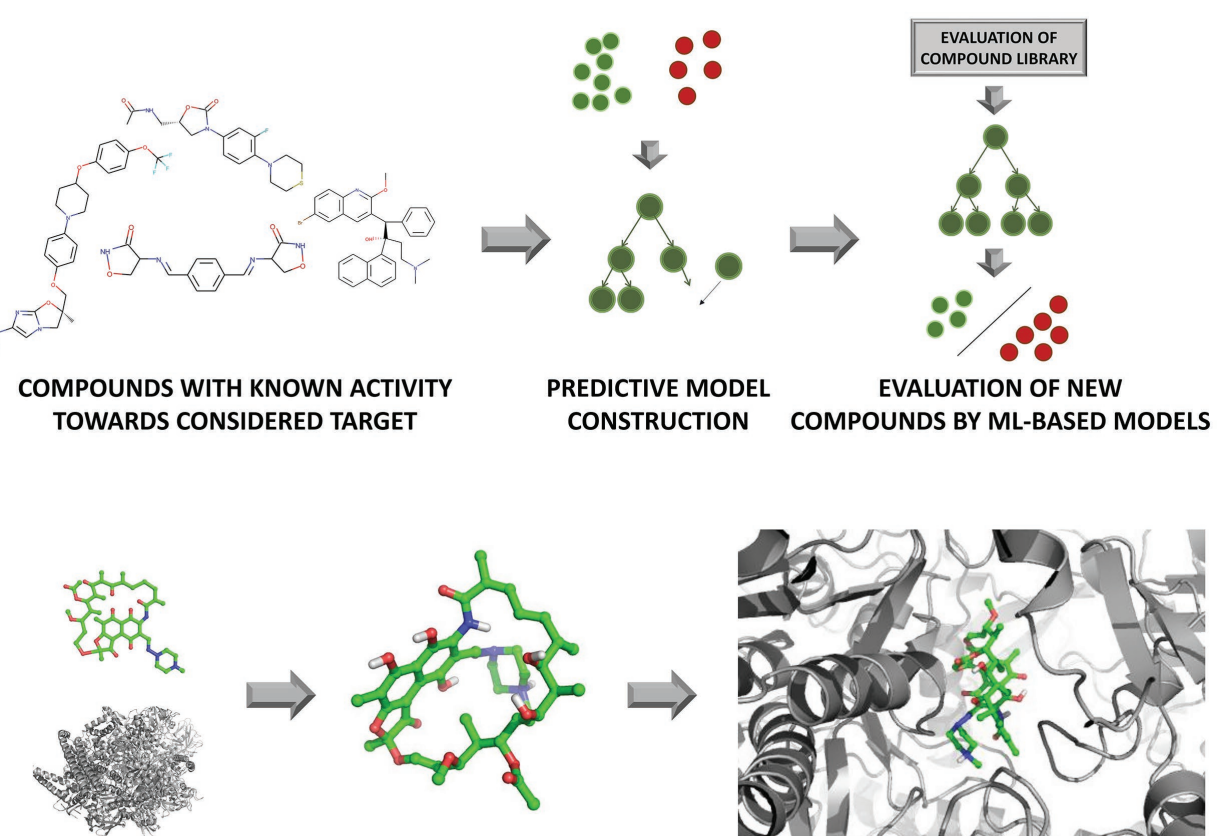

COMPOUND SET AND PROTEIN PREPARATION

GENERATION OF 3-DIMENSIONAL COMPOUND CONFORMATIONS

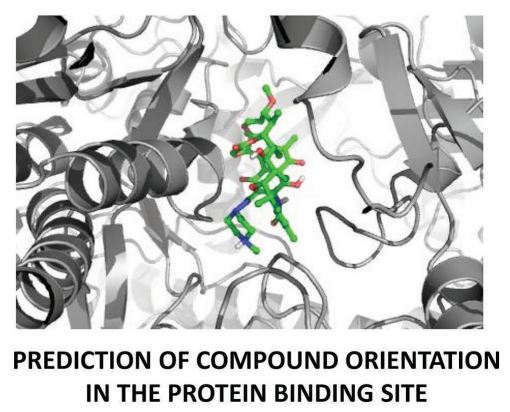

FIGURE 3 | In silico evaluation of compound activity with the use of (A) machine learning (ML) algorithms representing ligand-based approach, and (B) docking (element of the structure-based path).

Intensive growth of computational power on the one hand, and the increasing amount of data both in the ligandand structure-based field, has made simple statistical methods to be replaced with more complex models to analyze such data, with machine learning (ML) being on the top of the used methodologies (Figure 3). The main task of $\mathrm{ML}$ is to analyze existing data, and on their basis, construct a predictive model, which is then used for the evaluation of new examples (Mitchell, 2014).

Machine learning tools can be categorized into unsupervised (clustering methods) and supervised (regression, classifier analysis) learning. Both of these types are used in the search for new drugs, depending on the wanted outcome. ML is useful in all steps of the new drug discovery pipeline, especially in tasks where vast amounts of data need to be analyzed. It helps in the identification of new potential ligands in the VS procedure, generation sets of new potentially active compounds [deep learning, (DL)], optimization of compound physicochemical and ADMET properties, and detection of compound interactions with off-targets (Carpenter et al., 2018; Chen et al., 2018). ML is also useful in pre- and clinical development for cell response classification after drug intake (Vamathevan et al., 2019), as well as after the introduction of the drug to the market, e.g., for analyzing and monitoring the drug efficiency and possible side effects (Dimitri and Lió, 2017; Gao et al., 2017).

In an exemplary ML-based study to search for new antimycobacterial compounds, Prakash and colleagues built a database of compounds with known antitubercular effect, divided into three activity classes. Then, the compounds were clustered according to their chemical structure and four clusters acting on different targets were selected for further analysis. Cluster numbered 10 consisted of compounds, e.g., aminohydrazones, iso-nicotinoyl hydrazones, and iso-nicotinohydrazides that inhibit KatG and 2-trans-enoyl-acyl carrier protein reductase (InhA). Cluster 57 included pyrrole derivatives and azole antifungals, which interact with CYP51 isozymes. The next cluster contained oxazolidinones, which bind DNA gyrase, and 2-benzylthiopydidine-4-carbithioamide derivatives, which targets are not known. The last cluster contained pyridobenzoxaine derivatives of LFX and nitroquinolones. Hologram QSAR (HQSAR) allowed the search for fragments of molecules contributing to particular compound activity and to detect moieties that discriminated against active and inactive compounds. Individual active motifs found via this procedure were fused, and new bioactive motifs were proposed. Furthermore, they verified the effectiveness of new motifs by comparing them to compounds in the previously constructed dataset. One of the created motifs was found in already existing drugs: RMP, rifabutin, cirpofloxacin (COX), and ofloxacin (OX; Prakash and Ghosh, 2006).

Another similar approach used in drug design is searching for molecular patterns in other known drugs. In one of the early studies, researchers built their computational model by combining four linear equations and then apply it to screen the compounds found in Merck and Sigma-Aldrich catalogs. They selected 18 new compounds for microbial tests, which 
have not worked in their favor despite careful preparation of the model. ML models at that time were far from ideal. However, the study was able to pick four compounds that already have been experimentally checked for inhibiting $M$. tuberculosis growth, e.g., LZD, paromomycin, reserpine, and trifluoperazine from 5,000 compounds in the database, as well as compounds with new structures not found in currently used drugs treating TB (García-García et al., 2005). Some researchers are leaning toward Bayesian models since they are better suited for global QSAR analysis, can manage more data, and the results are easier to interpret and reproduce. The good performance of this model was proven by a study in which out of 44 antituberculars only six $(13,6 \%)$ were assigned to the wrong group based on chemical structure (Prathipati et al., 2008). In a similar study, Ekins and colleagues found through Bayesian modeling drugs that have not been yet experimentally verified against $M$. tuberculosis but scored high, e.g., sertaconazole, clofarabine, tioconazole, amodiaquine, quinaldine blue, atorvastatin, montelukast, daunorubicin, 4'-methoxychalcone, inosine, hieracin, iridin, harmane, and irigenol (Ekins and Freundlich, 2011).

Some researchers guided by the principles of polypharmacology are moving away from the "one target-one hit" model and looking into drugs that can potentially inhibit multiple targets (Zhang et al., 2016). The reasoning of this approach lies in the fact that treatment for TB already consists of multiple antimicrobials administered for a very long time (over 6 months for drug-susceptible M. tuberculosis; Tiberi et al., 2018a). Therefore drugs inhibiting multiple targets would significantly simplify treatment. There is also the possibility that adequate multi-target compounds will be more effective against drug-resistant $\mathrm{TB}$ and will not lead to the emergence of resistance as fast as one-target drugs do. Following this reasoning, Speck-Planche et al. (2012) created a model for mt-QSAR (multiple target QSAR). The difference between this method and QSAR was in the training dataset, which was constructed based on compounds active against all six proteins GyrA, GyrB, InhA, Ag85C, PS, and PD. Through combined VS, QSAR, and structure-based pharmacophore models, researchers found initial hits against InhA, GlmU, and DapB. Next, they added other known drug targets to create possible multi-target drugs (Janardhan et al., 2017). In another study focusing on phytochemicals through VS, researchers found four compounds amentoflavone, carpaine, 13-bromo-tiliacorinine and 2-nortiliacorinine, that bind with high affinity to many M. tuberculosis proteins, like Ask, DdIA, PanC, TrpB, AroF, NadE, AtpE, RibH, RpIE, and RpsE (Kumar et al., 2019). All results described above need yet to be confirmed by in vitro studies.

Since ML is used as a tool for finding new compounds through the mining of chemical databases, a number of databases gathering information on compound structure and bioactivity have been constructed. An example of such a database is a Collaborative Drug Discovery (CDD, Burlingame, CA), which now consists of more than 200,000 molecules (Hohman et al., 2009; Ekins et al., 2010). Since its construction, CDD was used in many important TB projects like the EU-funded New
Medicines 4 Tuberculosis (NM4TB) initiative (Ekins et al., 2011). Other popular databases of bioactive molecules are ChEMBL (which now contains almost 2 million distinct compounds, with over 16 million biological activities annotated) and PDSP with $\sim 10,000$ compound affinities toward different targets gathered (Besnard et al., 2012; Gaulton et al., 2012).

\section{CONSIDERATION OF DRUG RESISTANCE}

The principal molecular basis for mycobacterial diversity and drug resistance are single-nucleotide polymorphisms (SNPs). SNPs occur in the genome as a result of replication errors or erroneous DNA repair. M. tuberculosis lacks horizontal gene transfer trough mobile genetic elements such as plasmids or transposons. The comparison of the genomes of various $M$. tuberculosis strains revealed their similarity at over 99\% (Namouchi et al., 2012).

Many mutations encoding drug resistance are located in the direct drug targets of the proteins, e.g., rpoB (RMP; Telenti et al., 1993), embCAB operon (EMB; Mikusová et al., 1995; Telenti et al., 1997), rrs (KAN; Georghiou et al., 2012), gyrA, gyrB (fluoroquinolones, FQ; Takiff et al., 1994). However, drug resistance mutations are also associated with other loci. For example, in the case of INH, mutations occur in a seemingly unrelated $a h p C$ gene encoding alkyl hydroperoxidase It turns out that AhpC takes over the role of KatG, which is a catalaseperoxidase responsible for the transformation of INH from pro-drug to effective drug, in protecting the genome from oxygen-induced damage. The overexpression of AhpC significantly slows down the production of KatG. Thus, less INH is activated, and cells can survive (Sherman et al., 1999).

Mutations occurring in drug targets can negatively affect proteins metabolic activity, resulting in a deficit of cell fitness. Bacterial cells compensate through compensatory mutations. Mutants carrying compensatory mutations are characterized by lower fitness costs associated with drug resistance. The bestknown example of drug-resistant mutations causing fitness cost is RpoB (RMP resistance; Gagneux et al., 2006). Compensatory mutations were found in $\mathrm{RpoB}$ and other proteins of RNA polymerase complex, RpoA, and RpoC (Gagneux et al., 2006; Comas et al., 2012; de Vos et al., 2013).

\section{DNA Sequencing}

The principal identification of drug resistance sources is based on the cultivation of bacteria with antibiotics until drug-resistant variants appear (Takiff et al., 1994; Telenti et al., 1997). The genome sequences of drug-resistant clones are sequenced and screened for mutations (Telenti et al., 1997). This approach was used to identify drug-resistant mutations for major antitubercular drugs like EMB (Telenti et al., 1997) and FQ (Takiff et al., 1994). Notably, the amount of drug-resistant variants that can be detected in such studies is limited. As a high-throughput method, TraSH can be used to identify genes that do not sustain insertion among the pool of mutants 
(Sassetti et al., 2003; van Opijnen et al., 2009). Researchers tested the sensitivity of 69 morphotype mutants of M. smegmatis to one of the commonly used antibiotics - ampicillin to identify cell envelope genes associated with B-lactam resistance. After receiving four sensitive mutants, the transposon insertion sites were mapped (Viswanathan et al., 2017).

\section{Transcriptomics and Genetic Modification of Bacteria}

Changes in gene expression profiles studied through RNA-seq allow understanding of antibiotics' effect on M. tuberculosis physiology regarding tolerance mechanisms and drug resistance (Jain et al., 2016; Briffotaux et al., 2019). For example, increased the expression of the efpA gene encoding the efflux pump from the MFS family (major facilitator superfamily) and the iniA gene encoding one of the putative components of the efflux pump, after treatment with INH, may indicate that microorganisms are acquiring resistance (Briffotaux et al., 2019). Exposition of M. tuberculosis to antibiotics results in the overexpression of genes encoding DNA repair proteins (Gorna et al., 2010), e.g., dinX after RMP treatment (Boshoff et al., 2004), ssb after CM treatment (Fu and Shinnick, 2007), ada, alkA after treatment LFX (Boshoff et al., 2004) or xthA after treatment with OX (Boshoff et al., 2004) and many others.

The induction of efflux pumps can be visualized with reporter systems. Jain et al. (2016) investigated the gene expression profile in the presence of a subinhibitory concentration of INH. The addition of INH to $M$. tuberculosis during the logarithmic growth phase caused a CFU decrease of 2-3 logs. Mycobacterial cells that survived the treatment were characterized by an increased expression of specific genes, which indicate the formation of persister cells. These gene promoters were fused to a gene encoding the red fluorescent protein to create a reporter system for persisters.

\section{Bioinformatic Predictions}

Genome-wide association studies (GWAS) identify drug resistance sources after the drugs are already on the market. GWAS utilize genomic DNA sequencing data and statistics to study the association between gene variants across the population and the phenotypic traits, for example, variants of genes giving rise to drug-resistant strains (Power et al., 2017). Importantly, GWAS studies allow the identification of rare drug resistance variants or low-level drug resistance variants. GWAS of Farhat et al. (2013) study showed that potentially noncoding regions of the genome, like promoters of genes, contribute to drug resistance. The same team has confirmed noncoding regions associated with drug resistance for INH, EMB, and PZA. However, their effect on drug MIC was smaller than the effect of coding region mutations (Farhat et al., 2019). In subsequent studies, Coll et al. (2018) used GWAS to identify drug-resistance associated mutations in 6465 clinical strains. Most of the mutations and loci they found were well-known, but some of them were new, including loci in foliC, ubiA, thyX-hsdS.1, thy A, alr, ald, and $d f r A-t h y \mathrm{~A}$. They also found mutations in ethA and thy $\mathrm{X}$ promoters that may contribute to resistance emergence. GWAS is also used for studying characteristics of populations of M. tuberculosis. Oppong et al. (2019) through GWAS analysis, examined whether phylogenetic lineage background impacts drug resistance and found that particular drug resistance specific loci occur only in selected lineages.

The development of -omic technologies and computational power allowed the development of biological network models. These models are based on systemic biology, which combines knowledge about organisms on all organizational levels. The models take into account all the complex interactions and mechanisms occurring in the cells. There are different types of such networks, e.g., transcription factors-binding, PIP networks, metabolic interaction networks, genetic interaction networks, and others (Zhu et al., 2007; Chandra et al., 2011; Chung et al., 2013).

In silico analyses like PIP are applicable in predicting drug resistance patterns through building networks based on the STRING database and defining proteins that are drug targets and possible components of resistance emergence. Through this research, the concept of "co-target" was created. Co-target is a protein used simultaneously as a primary bacterial growth inhibitor to stop the emergence of resistance by affecting proteins in the resistance emergence pathway. These co-targets can be proteins associated with SOS response (RecA, RuvA, and LexA), involved in HGT (SecA1 and SecA2) or metabolism of drugs inside the cell like cytochromes and degrading enzymes (Cyp135, Erm37), but also proteins involved in the transportation of drugs out of the cell through efflux pumps (PstB; Raman and Chandra, 2008). Besides, PIP networks allow for learning more about the effects of antibiotics on the cells by finding what pathways are set in motion when antibiotics kill bacteria (Kohanski et al., 2010).

Identification of rare drug resistance mutations can also be made through ML. Based on 1983 M. tuberculosis isolates, Yang et al. (2018) developed ML models for four first-line drugs - INH, RMP, EMB, PZA, and several second-line drugs that analyze WGS data. The models increased the sensitivity of the detection of drug resistance when compared with previous studies. For EMB and RMP, the sensitivity increased to $97 \%$ $(p<0.01)$, and for COX and multi-drug resistant TB, it increased to $96 \%$. The INH had the lowest results, with only a $2-4 \%$ increase. In another study, Deelder et al. (2019) utilized WGS data to compare GWAS and their ML model. Both methods reached similar results, but GWAS was slightly more accurate for CM, CS, and KAN.

In the best scenario, mutations associated with drugresistance are confirmed through obtaining genetic mutants and observing the resulting drug-resistant phenotype. Another approach to confirm that a mutation is linked to drug resistance is through in silico docking. In silico docking is a useful tool for predicting the roles of mutations causing drug resistance. It can be done by analyzing interactions between the selected drug and protein, the wild-type, and the mutated variant (Nachappa et al., 2020). 


\section{ENTERING THE PRECLINICAL STAGE}

Researchers thoroughly check compounds with the potential to become drugs during the early discovery stage. Regardless of their usefulness from a biological point of view, novel compounds should have form facilitating administration. They have to go through tests of solubility, stability, and reactivity (Strovel et al., 2004; Hughes et al., 2011). If they fail to obtain satisfactory parameters, they are discarded. If they reach a satisfactory biological safety level and are practical, they proceed to the preclinical drug discovery stage. Preclinical studies are a checkpoint before human administration, and thus they are vital and carried with much caution.

Most of the compounds going through a hit to lead, and lead optimization, do not enter the preclinical stage. If we count all of the structures considered in chemical databases for screening, millions of compounds do not make it to the preclinical stage. Therefore the preclinical stage is often less costly than the early discovery stage. Up to our knowledge, there are no recent estimates on how many compounds go from the preclinical stage to the clinical phase. A common statement found in the articles is that out of 5,000 compounds entering the preclinical stage, five make it to the clinical stage, and then one makes it to the market (Kraljevic et al., 2004). Taken how many compounds undergo tests, the models for early drug discovery need to efficiently investigate broad biological consequences in a short time and at a low-cost.

\section{REFERENCES}

(2009). Screening we can believe in. Nat. Chem. Biol. 5:127. doi: 10.1038/ nchembio0309-127

Abrahams, G. L., Kumar, A., Savvi, S., Hung, A. W., Wen, S., Abell, C., et al. (2012). Pathway-selective sensitization of Mycobacterium tuberculosis for target-based whole-cell screening. Chem. Biol. 19, 844-854. doi: 10.1016/j. chembiol.2012.05.020

Adeniji, S. E., Uba, S., and Uzairu, A. (2018). QSAR modeling and molecular docking analysis of some active compounds against Mycobacterium tuberculosis receptor (Mtb CYP121). J. Pathog. 2018:e1018694. doi: 10.1155/2018/1018694

Ahmed, S., Raqib, R., Guðmundsson, G. H., Bergman, P., Agerberth, B., and Rekha, R. S. (2020). Host-directed therapy as a novel treatment strategy to overcome tuberculosis: targeting immune modulation. Antibiotics 9:21. doi: 10.3390/antibiotics9010021

Alksne, L. E., and Dunman, P. M. (2008). "Target-based antimicrobial drug discovery" in Bacterial pathogenesis. eds. F. R. DeLeo and M. Otto (Totowa, NJ: Humana Press), 271-283.

Altaf, M., Miller, C. H., Bellows, D. S., and O’Toole, R. (2010). Evaluation of the Mycobacterium smegmatis and BCG models for the discovery of Mycobacterium tuberculosis inhibitors. Tuberculosis 90, 333-337. doi: 10.1016/j. tube.2010.09.002

Amir, A., Rana, K., Arya, A., Kapoor, N., Kumar, H., and Siddiqui, M. A. (2014). Mycobacterium tuberculosis H37Rv: in silico drug targets identification by metabolic pathways analysis. Int. J. Evol. Biol. 2014:284170. doi: $10.1155 / 2014 / 284170$

Andries, K., Verhasselt, P., Guillemont, J., Göhlmann, H. W. H., Neefs, J. -M., Winkler, H., et al. (2005). A diarylquinoline drug active on the ATP synthase of Mycobacterium tuberculosis. Science 307, 223-227. doi: 10.1126/science. 1106753

Bahuguna, A., and Rawat, D. S. (2020). An overview of new antitubercular drugs, drug candidates, and their targets. Med. Res. Rev. 40, 263-292. doi: $10.1002 /$ med.21602

Balganesh, M., Dinesh, N., Sharma, S., Kuruppath, S., Nair, A. V., and Sharma, U. (2012). Efflux pumps of Mycobacterium tuberculosis play a significant role

\section{CONCLUSION}

The growing demand for discovering new antibiotics stimulates the constant development of research methods that broaden the knowledge about biological processes occurring in bacterial cells undergoing chemotherapy. Understanding these processes enables more effective identification of antitubercular compounds. Thanks to the -omics technologies, these compounds can now be safer and less prone to the generation of drug resistance. When combined, the -omics technologies allow us to gain a more holistic view of drug utility.

\section{AUTHOR CONTRIBUTIONS}

AM and JD designed the manuscript. AM, LŻ, EL, FG, AK, SP, DZ, and JD wrote the manuscript. All authors contributed to the article and approved the submitted version.

\section{FUNDING}

This work is part of the research project financed by the National Science Center of Poland, grant number 2019/34/E/ NZ6/00221.

in antituberculosis activity of potential drug candidates. Antimicrob. Agents Chemother. 56, 2643-2651. doi: 10.1128/AAC.06003-11

Besnard, J., Ruda, G. F., Setola, V., Abecassis, K., Rodriguiz, R. M., Huang, X. -P., et al. (2012). Automated design of ligands to polypharmacological profiles. Nature 492, 215-220. doi: 10.1038/nature11691

Betts, J. C., McLaren, A., Lennon, M. G., Kelly, F. M., Lukey, P. T., Blakemore, S. J., et al. (2003). Signature gene expression profiles discriminate between isoniazid-, thiolactomycin-, and triclosan-treated Mycobacterium tuberculosis. Antimicrob. Agents Chemother. 47, 2903-2913. doi: 10.1128/AAC.47.9.2903-2913.2003

Boot, M., Commandeur, S., Subudhi, A. K., Bahira, M., Smith, T. C., Abdallah, A. M., et al. (2018). Accelerating early antituberculosis drug discovery by creating mycobacterial indicator strains that predict mode of action. Antimicrob. Agents Chemother. 62:e00083-18. doi: 10.1128/AAC. 00083-18

Boshoff, H. I. M., Myers, T. G., Copp, B. R., McNeil, M. R., Wilson, M. A., and Barry, C. E. (2004). The transcriptional responses of Mycobacterium tuberculosis to inhibitors of metabolism: novel insights into drug mechanisms of action. J. Biol. Chem. 279, 40174-40184. doi: 10.1074/jbc.M406796200

Briffotaux, J., Liu, S., and Gicquel, B. (2019). Genome-wide transcriptional responses of Mycobacterium to antibiotics. Front. Microbiol. 10:249. doi: 10.3389/fmicb.2019.00249

Bryk, R., Gold, B., Venugopal, A., Singh, J., Samy, R., Pupek, K., et al. (2008). Selective killing of nonreplicating mycobacteria. Cell Host Microbe 3, 137-145. doi: 10.1016/j.chom.2008.02.003

Campaniço, A., Moreira, R., and Lopes, F. (2018). Drug discovery in tuberculosis. New drug targets and antimycobacterial agents. Eur. J. Med. Chem. 150, 525-545. doi: 10.1016/j.ejmech.2018.03.020

Carey, A. F., Rock, J. M., Krieger, I. V., Chase, M. R., Fernandez-Suarez, M., Gagneux, S., et al. (2018). TnSeq of Mycobacterium tuberculosis clinical isolates reveals strain-specific antibiotic liabilities. PLoS Pathog. 14:e1006939. doi: 10.1371/journal.ppat.1006939

Carpenter, K. A., Cohen, D. S., Jarrell, J. T., and Huang, X. (2018). Deep learning and virtual drug screening. Future Med. Chem. 10, 2557-2567. doi: $10.4155 /$ fmc-2018-0314

Chandra, N., Kumar, D., and Rao, K. (2011). Systems biology of tuberculosis. Tuberculosis 91, 487-496. doi: 10.1016/j.tube.2011.02.008 
Chatterjee, A., Sharma, A. K., Mahatha, A. C., Banerjee, S. K., Kumar, M., Saha, S., et al. (2018). Global mapping of MtrA-binding sites links MtrA to regulation of its targets in Mycobacterium tuberculosis. Microbiology 164, 99-110. doi: 10.1099/mic.0.000585

Chen, H., Engkvist, O., Wang, Y., Olivecrona, M., and Blaschke, T. (2018). The rise of deep learning in drug discovery. Drug Discov. Today 23, 1241-1250. doi: 10.1016/j.drudis.2018.01.039

Choudhary, E., Thakur, P., Pareek, M., and Agarwal, N. (2015). Gene silencing by CRISPR interference in mycobacteria. Nat. Commun. 6:6267. doi: 10.1038/ ncomms 7267

Chung, B. K. -S., Dick, T., and Lee, D. -Y. (2013). In silico analyses for the discovery of tuberculosis drug targets. J. Antimicrob. Chemother. 68, 2701-2709. doi: $10.1093 / \mathrm{jac} / \mathrm{dkt} 273$

Coll, F., Phelan, J., Hill-Cawthorne, G. A., Nair, M. B., Mallard, K., Ali, S., et al. (2018). Genome-wide analysis of multi- and extensively drug-resistant Mycobacterium tuberculosis. Nat. Genet. 50, 307-316. doi: 10.1038/s41588017-0029-0

Comas, I., Borrell, S., Roetzer, A., Rose, G., Malla, B., Kato-Maeda, M., et al. (2012). Whole-genome sequencing of rifampicin-resistant Mycobacterium tuberculosis strains identifies compensatory mutations in RNA polymerase genes. Nat. Genet. 44, 106-110. doi: 10.1038/ng.1038

Dara, Y., Volcani, D., Shah, K., Shin, K., and Venketaraman, V. (2019). Potentials of host-directed therapies in tuberculosis management. J. Clin. Med. 8:1166. doi: $10.3390 / \mathrm{jcm} 8081166$

Das, N., Jena, P. K., and Pradhan, S. K. (2020). Arabinosyltransferase C enzyme of Mycobacterium tuberculosis, a potential drug target: an insight from molecular docking study. Heliyon 6:e02693. doi: 10.1016/j.heliyon.2019.e02693

de Carvalho, L. P. S., Fischer, S. M., Marrero, J., Nathan, C., Ehrt, S., and Rhee, K. Y. (2010). Metabolomics of Mycobacterium tuberculosis reveals compartmentalized co-catabolism of carbon substrates. Chem. Biol. 17, 1122-1131. doi: 10.1016/j.chembiol.2010.08.009

de Vos, M., Müller, B., Borrell, S., Black, P. A., van Helden, P. D., Warren, R. M., et al. (2013). Putative compensatory mutations in the rpoC gene of rifampinresistant Mycobacterium tuberculosis are associated with ongoing transmission. Antimicrob. Agents Chemother. 57, 827-832. doi: 10.1128/AAC.01541-12

Deelder, W., Christakoudi, S., Phelan, J., Benavente, E. D., Campino, S., McNerney, R., et al. (2019). Machine learning predicts accurately Mycobacterium tuberculosis drug resistance from whole genome sequencing data. Front. Genet. 10:922. doi: 10.3389/fgene.2019.00922

DeJesus, M. A., Gerrick, E. R., Xu, W., Park, S. W., Long, J. E., Boutte, C. C., et al. (2017). Comprehensive essentiality analysis of the Mycobacterium tuberculosis genome via saturating transposon mutagenesis. MBio 8:e02133-16. doi: $10.1128 / \mathrm{mBio} .02133-16$

Deoghare, S. (2013). Bedaquiline: a new drug approved for treatment of multidrug-resistant tuberculosis. Indian J. Pharm. 45, 536-537. doi: 10.4103/ 0253-7613.117765

Dimitri, G. M., and Lió, P. (2017). DrugClust: a machine learning approach for drugs side effects prediction. Comput. Biol. Chem. 68, 204-210. doi: 10.1016/j.compbiolchem.2017.03.008

Dooley, K. E., Hanna, D., Mave, V., Eisenach, K., and Savic, R. M. (2019). Advancing the development of new tuberculosis treatment regimens: the essential role of translational and clinical pharmacology and microbiology. PLoS Med. 16:e1002842. doi: 10.1371/journal.pmed.1002842

Dutta, N. K., Mehra, S., Didier, P. J., Roy, C. J., Doyle, L. A., Alvarez, X., et al. (2010). Genetic requirements for the survival of tubercle bacilli in primates. J. Infect. Dis. 201, 1743-1752. doi: 10.1086/652497

Ekins, S., Bradford, J., Dole, K., Spektor, A., Gregory, K., Blondeau, D., et al. (2010). A collaborative database and computational models for tuberculosis drug discovery. Mol. BioSyst. 6, 840-851. doi: 10.1039/B917766C

Ekins, S., and Freundlich, J. S. (2011). Validating new tuberculosis computational models with public whole cell screening aerobic activity datasets. Pharm. Res. 28, 1859-1869. doi: 10.1007/s11095-011-0413-x

Ekins, S., Freundlich, J. S., Choi, I., Sarker, M., and Talcott, C. (2011). Computational databases, pathway and cheminformatics tools for tuberculosis drug discovery. Trends Microbiol. 19, 65-74. doi: 10.1016/j.tim.2010.10.005

Esposito, M., Szadocka, S., Degiacomi, G., Orena, B. S., Mori, G., Piano, V., et al. (2017). A phenotypic based target screening approach delivers new antitubercular CTP synthetase inhibitors. ACS Infect. Dis. 3, 428-437. doi: 10.1021/acsinfecdis.7b00006
Farhat, M. R., Freschi, L., Calderon, R., Ioerger, T., Snyder, M., Meehan, C. J., et al. (2019). GWAS for quantitative resistance phenotypes in Mycobacterium tuberculosis reveals resistance genes and regulatory regions. Nat. Commun. 10:2128. doi: 10.1038/s41467-019-10110-6

Farhat, M. R., Shapiro, B. J., Kieser, K. J., Sultana, R., Jacobson, K. R., Victor, T. C., et al. (2013). Genomic analysis identifies targets of convergent positive selection in drug-resistant Mycobacterium tuberculosis. Nat. Genet. 45, 1183-1189. doi: 10.1038/ng.2747

Fields, F. R., Lee, S. W., and McConnell, M. J. (2017). Using bacterial genomes and essential genes for the development of new antibiotics. Biochem. Pharmacol. 134, 74-86. doi: 10.1016/j.bcp.2016.12.002

Fu, L. M., and Shinnick, T. M. (2007). Genome-wide exploration of the drug action of capreomycin on Mycobacterium tuberculosis using Affymetrix oligonucleotide GeneChips. J. Inf. Secur. 54, 277-284. doi: 10.1016/j.jinf. 2006.05.012

Gagneux, S., Long, C. D., Small, P. M., Van, T., Schoolnik, G. K., and Bohannan, B. J. M. (2006). The competitive cost of antibiotic resistance in Mycobacterium tuberculosis. Science 312, 1944-1946. doi: 10.1126/science. 1124410

Gao, M., Igata, H., Takeuchi, A., Sato, K., and Ikegaya, Y. (2017). Machine learning-based prediction of adverse drug effects: an example of seizureinducing compounds. J. Pharmacol. Sci. 133, 70-78. doi: 10.1016/j.jphs. 2017.01.003

García-García, A., Gálvez, J., de Julián-Ortiz, J. V., García-Domenech, R., Muñoz, C., Guna, R., et al. (2005). Search of chemical scaffolds for novel antituberculosis agents. J. Biomol. Screen. 10, 206-214. doi: 10.1177/108705 7104273486

Gaulton, A., Bellis, L. J., Bento, A. P., Chambers, J., Davies, M., Hersey, A., et al. (2012). ChEMBL: a large-scale bioactivity database for drug discovery. Nucleic Acids Res. 40, D1100-D1107. doi: 10.1093/nar/gkr777

Georghiou, S. B., Magana, M., Garfein, R. S., Catanzaro, D. G., Catanzaro, A., and Rodwell, T. C. (2012). Evaluation of genetic mutations associated with Mycobacterium tuberculosis resistance to amikacin, kanamycin and capreomycin: a systematic review. PLoS One 7:e33275. doi: 10.1371/journal.pone.0033275

Ghajavand, H., Kamakoli, M. K., Khanipour, S., Dizaji, S. P., Masoumi, M., Jamnani, F. R., et al. (2019). High prevalence of bedaquiline resistance in treatment-naive tuberculosis patients and verapamil effectiveness. Antimicrob. Agents Chemother. 63, e02530-e02618. doi: 10.1128/AAC.02530-18

Goff, A., Cantillon, D., Muraro Wildner, L., and Waddell, S. J. (2020). Multiomics technologies applied to tuberculosis drug discovery. Appl. Sci. 10:4629. doi: 10.3390/app10134629

Gorla, P., Plocinska, R., Sarva, K., Satsangi, A. T., Pandeeti, E., Donnelly, R., et al. (2018). MtrA response regulator controls cell division and cell wall metabolism and affects susceptibility of mycobacteria to the first line antituberculosis drugs. Front. Microbiol. 9:2839. doi: 10.3389/fmicb.2018.02839

Gorna, A. E., Bowater, R. P., and Dziadek, J. (2010). DNA repair systems and the pathogenesis of Mycobacterium tuberculosis: varying activities at different stages of infection. Clin. Sci. 119, 187-202. doi: 10.1042/CS20100041

Grant, S. S., Kawate, T., Nag, P. P., Silvis, M. R., Gordon, K., Stanley, S. A., et al. (2013). Identification of novel inhibitors of nonreplicating Mycobacterium tuberculosis using a carbon starvation model. ACS Chem. Biol. 8, 2224-2234. doi: $10.1021 / \mathrm{cb} 4004817$

Griffiths, W. J., and Wang, Y. (2009). Mass spectrometry: from proteomics to metabolomics and lipidomics. Chem. Soc. Rev. 38, 1882-1896. doi: 10.1039/ b618553n

Grzelak, E. M., Choules, M. P., Gao, W., Cai, G., Wan, B., Wang, Y., et al. (2019). Strategies in anti-Mycobacterium tuberculosis drug discovery based on phenotypic screening. J. Antibiot. 72, 719-728. doi: 10.1038/s41429-0190205-9

Gupta, A., and Bhakta, S. (2012). An integrated surrogate model for screening of drugs against Mycobacterium tuberculosis. J. Antimicrob. Chemother. 67, 1380-1391. doi: 10.1093/jac/dks056

Halouska, S., Fenton, R. J., Barletta, R. G., and Powers, R. (2012). Predicting the in vivo mechanism of action for drug leads using NMR metabolomics. ACS Chem. Biol. 7, 166-171. doi: 10.1021/cb200348m

Hernandez-Abanto, S. M., Cheng, Q. -J., Singh, P., Ly, L. H., Klinkenberg, L. G., Morrison, N. E., et al. (2007). Accelerated detection of Mycobacterium tuberculosis genes essential for bacterial survival in Guinea pigs, compared with mice. J. Infect. Dis. 195, 1634-1642. doi: 10.1086/517526 
Hohman, M., Gregory, K., Chibale, K., Smith, P. J., Ekins, S., and Bunin, B. (2009). Novel web-based tools combining chemistry informatics, biology and social networks for drug discovery. Drug Discov. Today 14, 261-270. doi: 10.1016/j.drudis.2008.11.015

Hu, Y., Liu, A., Menendez, M. C., Garcia, M. J., Oravcova, K., Gillespie, S. H., et al. (2015). HspX knock-out in Mycobacterium tuberculosis leads to shorter antibiotic treatment and lower relapse rate in a mouse model-a potential novel therapeutic target. Tuberculosis 95, 31-36. doi: 10.1016/j.tube.2014.11.002

Hughes, J. P., Rees, S., Kalindjian, S. B., and Philpott, K. L. (2011). Principles of early drug discovery. Br. J. Pharmacol. 162, 1239-1249. doi: 10.1111/j.1476-5381.2010.01127.x

Jagadeb, M., Rath, S. N., and Sonawane, A. (2019). In silico discovery of potential drug molecules to improve the treatment of isoniazid-resistant Mycobacterium tuberculosis. J. Biomol. Struct. Dyn. 37, 3388-3398. doi: 10.1080/07391102.2018.1515116

Jain, P., Weinrick, B. C., Kalivoda, E. J., Yang, H., Munsamy, V., Vilcheze, C., et al. (2016). Dual-reporter mycobacteriophages ( 2 2DRMs) reveal preexisting Mycobacterium tuberculosis persistent cells in human sputum. MBio 7:e1023-16. doi: 10.1128/mBio.01023-16

Janardhan, S., John, L., Prasanthi, M., Poroikov, V., and Narahari Sastry, G. (2017). A QSAR and molecular modelling study towards new lead finding: polypharmacological approach to Mycobacterium tuberculosis. SAR QSAR Environ. Res. 28, 815-832. doi: 10.1080/1062936X.2017.1398782

Kana, B. D., Karakousis, P. C., Parish, T., and Dick, T. (2014). Future targetbased drug discovery for tuberculosis? Tuberculosis 94, 551-556. doi: 10.1016/j. tube.2014.10.003

Kar, S., and Roy, K. (2013). How far can virtual screening take us in drug discovery? Expert Opin. Drug Discovery 8, 245-261. doi: 10.1517/174604 41.2013.761204

Kaufmann, S. H. E. (2020). Vaccination against tuberculosis: revamping BCG by molecular genetics guided by immunology. Front. Immunol. 11:316. doi: 10.3389/fimmu.2020.00316

Kohanski, M. A., Dwyer, D. J., and Collins, J. J. (2010). How antibiotics kill bacteria: from targets to networks. Nat. Rev. Microbiol. 8, 423-435. doi: $10.1038 /$ nrmicro2333

Korycka-Machala, M., Nowosielski, M., Kuron, A., Rykowski, S., Olejniczak, A., Hoffmann, M., et al. (2017). Naphthalimides selectively inhibit the activity of bacterial, replicative DNA ligases and display bactericidal effects against tubercle bacilli. Molecules 22:154. doi: 10.3390/molecules22010154

Korycka-Machała, M., Viljoen, A., Pawełczyk, J., Borówka, P., Dziadek, B., Gobis, K., et al. (2019). 1H-Benzo[d]imidazole derivatives affect MmpL3 in Mycobacterium tuberculosis. Antimicrob. Agents Chemother. 63, e00441-e00519. doi: 10.1128/AAC.00441-19

Koul, A., Vranckx, L., Dendouga, N., Balemans, W., Van den Wyngaert, I., Vergauwen, K., et al. (2008). Diarylquinolines are bactericidal for dormant mycobacteria as a result of disturbed ATP homeostasis. J. Biol. Chem. 283, 25273-25280. doi: 10.1074/jbc.M803899200

Kraljevic, S., Stambrook, P. J., and Pavelic, K. (2004). Accelerating drug discovery. EMBO Rep. 5, 837-842. doi: 10.1038/sj.embor.7400236

Kumar, A., Guardia, A., Colmenarejo, G., Pérez, E., Gonzalez, R. R., Torres, P., et al. (2015). A focused screen identifies antifolates with activity on Mycobacterium tuberculosis. ACS Infect. Dis. 1, 604-614. doi: 10.1021/ acsinfecdis. 5 b00063

Kumar, S., Sahu, P., and Jena, L. (2019). An in silico approach to identify potential inhibitors against multiple drug targets of Mycobacterium tuberculosis. Int. J. Mycobacteriol. 8, 252-261. doi: 10.4103/ijmy.ijmy_109_19

Kushwaha, S. K., and Shakya, M. (2010). Protein interaction network analysisapproach for potential drug target identification in Mycobacterium tuberculosis. J. Theor. Biol. 262, 284-294. doi: 10.1016/j.jtbi.2009.09.029

Kwan, P. K. W., Lin, W., Naim, A. N. M., Periaswamy, B., De Sessions, P. F., Hibberd, M. L., et al. (2020). Gene expression responses to anti-tuberculous drugs in a whole blood model. BMC Microbiol. 20:81. doi: 10.1186/ s12866-020-01766-y

Lee, J. Y. (2015). Diagnosis and treatment of extrapulmonary tuberculosis. Tuberc. Respir. Dis. 78, 47-55. doi: 10.4046/trd.2015.78.2.47

Lelovic, N., Mitachi, K., Yang, J., Lemieux, M. R., Ji, Y., and Kurosu, M. (2020). Application of Mycobacterium smegmatis as a surrogate to evaluate drug leads against Mycobacterium tuberculosis. J. Antibiot. 73, 780-789. doi: 10.1038/ s41429-020-0320-7
Lushington, G., and Chaguturu, R. (2014). To screen or not to screen: an impassioned plea for smarter chemical libraries to improve drug lead finding. Future Med. Chem. 6, 497-502. doi: 10.4155/fmc.14.21

Ma, S., Jaipalli, S., Larkins-Ford, J., Lohmiller, J., Aldridge, B. B., Sherman, D. R., et al. (2019). Transcriptomic signatures predict regulators of drug synergy and clinical regimen efficacy against tuberculosis. MBio 10:e02627-19. doi: 10.1128/mBio.02627-19

Macalino, S. J. Y., Billones, J. B., Organo, V. G., and Carrillo, M. C. O. (2020). In silico strategies in tuberculosis drug discovery. Molecules 25:665. doi: 10.3390/molecules25030665

Mallavarapu, B. D., Abdullah, M., Saxena, S., and Guruprasad, L. (2019). Inhibitor binding studies of Mycobacterium tuberculosis MraY (Rv2156c): insights from molecular modeling, docking, and simulation studies. J. Biomol. Struct. Dyn. 37, 3751-3763. doi: 10.1080/07391102.2018.1526715

Martin, Y. C., Kofron, J. L., and Traphagen, L. M. (2002). Do structurally similar molecules have similar biological activity? J. Med. Chem. 45, 4350-4358. doi: $10.1021 / \mathrm{jm} 020155 \mathrm{c}$

McNeil, M. B., and Cook, G. M. (2019). Utilization of CRISPR interference to validate MmpL3 as a drug target in Mycobacterium tuberculosis. Antimicrob. Agents Chemother. 63, e00629-e00719. doi: 10.1128/AAC.00629-19

Melak, T., and Gakkhar, S. (2014). Potential non homologous protein targets of Mycobacterium tuberculosis $\mathrm{H} 37 \mathrm{Rv}$ identified from protein-protein interaction network. J. Theor. Biol. 361, 152-158. doi: 10.1016/j.jtbi. 2014.07.031

Melak, T., and Gakkhar, S. (2015). Comparative genome and network centrality analysis to identify drug targets of Mycobacterium tuberculosis H37Rv. Biomed. Res. Int. 2015:212061. doi: 10.1155/2015/212061

Mikusová, K., Slayden, R. A., Besra, G. S., and Brennan, P. J. (1995). Biogenesis of the mycobacterial cell wall and the site of action of ethambutol. Antimicrob. Agents Chemother. 39, 2484-2489. doi: 10.1128/aac.39.11.2484

Minias, A., Brzostek, A., and Dziadek, J. (2019). Targeting DNA repair systems in antitubercular drug development. Curr. Med. Chem. 26, 1494-1505 doi: 10.2174/0929867325666180129093546

Mitchell, J. B. O. (2014). Machine learning methods in chemoinformatics. Wiley Interdiscip. Rev. Comput. Mol. Sci. 4, 468-481. doi: 10.1002/ wcms. 1183

Muliaditan, M., Davies, G. R., Simonsson, U. S. H., Gillespie, S. H., and Della Pasqua, O. (2017). The implications of model-informed drug discovery and development for tuberculosis. Drug Discov. Today 22, 481-486. doi: 10.1016/j. drudis.2016.09.004

Murray, J. F., Schraufnagel, D. E., and Hopewell, P. C. (2015). Treatment of tuberculosis. A historical perspective. Ann. Am. Thorac. Soc. 12, 1749-1759. doi: 10.1513/AnnalsATS.201509-632PS

Nachappa, S., Neelambike, S., Sarikhani, A., and Ramachandra, N. (2020). Simultaneous detection of drug-resistant mutations in Mycobacterium tuberculosis and determining their role through in silico docking. Infect. Disord. Drug Targets. doi: 10.2174/1871526520666200318111140 [Epub ahead of print]

Namouchi, A., Didelot, X., Schöck, U., Gicquel, B., and Rocha, E. P. C. (2012). After the bottleneck: genome-wide diversification of the Mycobacterium tuberculosis complex by mutation, recombination, and natural selection. Genome Res. 22, 721-734. doi: 10.1101/gr.129544.111

Nguyen, Q. H., Contamin, L., Nguyen, T. V. A., and Bañuls, A. (2018). Insights into the processes that drive the evolution of drug resistance in Mycobacterium tuberculosis. Evol. Appl. 11, 1498-1511. doi: 10.1111/eva.12654

Nowosielski, M., Hoffmann, M., Kuron, A., Korycka-Machala, M., and Dziadek, J. (2013). The MM2QM tool for combining docking, molecular dynamics, molecular mechanics, and quantum mechanics. J. Comput. Chem. 34, 750-756. doi: $10.1002 /$ jcc. 23192

O’Rourke, A., Beyhan, S., Choi, Y., Morales, P., Chan, A. P., Espinoza, J. L., et al. (2020). Mechanism-of-action classification of antibiotics by global transcriptome profiling. Antimicrob. Agents Chemother. 64, e01207-e01319. doi: 10.1128/AAC.01207-19

Oppong, Y. E. A., Phelan, J., Perdigão, J., Machado, D., Miranda, A., Portugal, I., et al. (2019). Genome-wide analysis of Mycobacterium tuberculosis polymorphisms reveals lineage-specific associations with drug resistance. BMC Genomics 20:252. doi: 10.1186/s12864-019-5615-3

Osterman, A. L., Rodionova, I., Li, X., Sergienko, E., Ma, C. -T., Catanzaro, A., et al. (2019). Novel antimycobacterial compounds suppress NAD biogenesis 
by targeting a unique pocket of NaMN adenylyltransferase. ACS Chem. Biol. 14, 949-958. doi: 10.1021/acschembio.9b00124

Pal, R., Hameed, S., Sabareesh, V., Kumar, P., Singh, S., and Fatima, Z. (2018). Investigations into isoniazid treated Mycobacterium tuberculosis by electrospray mass spectrometry reveals new insights into its lipid composition. J. Pathog. 2018:e1454316. doi: 10.1155/2018/1454316

Parish, T., and Stoker, N. G. (2000). Use of a flexible cassette method to generate a double unmarked Mycobacterium tuberculosis tlyA plcABC mutant by gene replacement. Microbiology 146, 1969-1975. doi: 10.1099/00221 287-146-8-1969

Płocinska, R., Korycka-Machala, M., Plocinski, P., and Dziadek, J. (2017). Mycobacterial DNA replication as a target for antituberculosis drug discovery. Curr. Top. Med. Chem. 17, 2129-2142. doi: 10.2174/156802661766 6170130114342

Plocinska, R., Purushotham, G., Sarva, K., Vadrevu, I. S., Pandeeti, E. V. P., Arora, N., et al. (2012). Septal localization of the Mycobacterium tuberculosis MtrB sensor kinase promotes MtrA regulon expression. J. Biol. Chem. 287, 23887-23899. doi: 10.1074/jbc.M112.346544

Power, R. A., Parkhill, J., and de Oliveira, T. (2017). Microbial genome-wide association studies: lessons from human GWAS. Nat. Rev. Genet. 18, 41-50. doi: $10.1038 / \mathrm{nrg} .2016 .132$

Prakash, O., and Ghosh, I. (2006). Developing an antituberculosis compounds database and data mining in the search of a motif responsible for the activity of a diverse class of antituberculosis agents. J. Chem. Inf. Model. 46, 17-23. doi: 10.1021/ci050115s

Prathipati, P., Ma, N. L., and Keller, T. H. (2008). Global Bayesian models for the prioritization of antitubercular agents. J. Chem. Inf. Model. 48, 2362-2370. doi: $10.1021 /$ ci800143n

Prosser, G. A., and de Carvalho, L. P. S. (2013). Metabolomics reveal d-alanine:dalanine ligase as the target of d-cycloserine in Mycobacterium tuberculosis. ACS Med. Chem. Lett. 4, 1233-1237. doi: 10.1021/ml400349n

Raghunandanan, S., Jose, L., Gopinath, V., and Kumar, R. A. (2019). Comparative label-free lipidomic analysis of Mycobacterium tuberculosis during dormancy and reactivation. Sci. Rep. 9:3660. doi: 10.1038/s41598-019-40051-5

Raman, K., and Chandra, N. (2008). Mycobacterium tuberculosis interactome analysis unravels potential pathways to drug resistance. BMC Microbiol. 8:234. doi: 10.1186/1471-2180-8-234

Sassetti, C. M., Boyd, D. H., and Rubin, E. J. (2003). Genes required for mycobacterial growth defined by high density mutagenesis. Mol. Microbiol. 48, 77-84. doi: 10.1046/j.1365-2958.2003.03425.x

Sassetti, C. M., and Rubin, E. J. (2003). Genetic requirements for mycobacterial survival during infection. Proc. Natl. Acad. Sci. U. S. A. 100, 12989-12994. doi: 10.1073/pnas.2134250100

Schmidt, T., Bergner, A., and Schwede, T. (2014). Modelling three-dimensional protein structures for applications in drug design. Drug Discov. Today 19, 890-897. doi: 10.1016/j.drudis.2013.10.027

Sherman, D. R., Mdluli, K., Hickey, M. J., Barry, C. E., and Stover, C. K. (1999). AhpC, oxidative stress and drug resistance in Mycobacterium tuberculosis. Biofactors 10, 211-217. doi: 10.1002/biof.5520100219

Silver, L. L. (2012). "Rational approaches to antibacterial discovery: pregenomic directed and phenotypic screening" in Antibiotic discovery and development. eds. T. J. Dougherty and M. J. Pucci (Boston, MA: Springer US), 33-75.

Speck-Planche, A., Kleandrova, V. V., Luan, F., Natalia, D. S., and Cordeiro, M. (2012). In silico discovery and virtual screening of multi-target inhibitors for proteins in Mycobacterium tuberculosis. Comb. Chem. High Throughput Screen 15, 666-673. doi: 10.2174/138620712802650487.

Strovel, J., Sittampalam, S., Coussens, N. P., Hughes, M., Inglese, J., Kurtz, A., et al. (2004). "Early drug discovery and development guidelines: for academic researchers, collaborators, and start-up companies" in Assay guidance manual. eds. S. Markossian, G. S. Sittampalam, A. Grossman, K. Brimacombe, M. Arkin and D. Auld et al. (Bethesda (MD): Eli Lilly \& Company and the National Center for Advancing Translational Sciences).

Syre, H., Phyu, S., Sandven, P., Bjorvatn, B., and Grewal, H. M. S. (2003). Rapid colorimetric method for testing susceptibility of Mycobacterium tuberculosis to isoniazid and rifampin in liquid cultures. J. Clin. Microbiol. 41, 5173-5177. doi: 10.1128/JCM.41.11.5173-5177.2003

Takiff, H. E., Salazar, L., Guerrero, C., Philipp, W., Huang, W. M., Kreiswirth, B., et al. (1994). Cloning and nucleotide sequence of Mycobacterium tuberculosis
gyrA and gyrB genes and detection of quinolone resistance mutations. Antimicrob. Agents Chemother. 38, 773-780. doi: 10.1128/aac.38.4.773

Telenti, A., Marchesi, F., Balz, M., Bally, F., Böttger, E. C., and Bodmer, T. (1993). Rapid identification of mycobacteria to the species level by polymerase chain reaction and restriction enzyme analysis. J. Clin. Microbiol. 31, 175-178. doi: 10.1128/JCM.31.2.175-178.1993

Telenti, A., Philipp, W. J., Sreevatsan, S., Bernasconi, C., Stockbauer, K. E., Wieles, B., et al. (1997). The emb operon, a gene cluster of Mycobacterium tuberculosis involved in resistance to ethambutol. Nat. Med. 3, 567-570. doi: 10.1038/nm0597-567

Theuretzbacher, U., Outterson, K., Engel, A., and Karlén, A. (2020). The global preclinical antibacterial pipeline. Nat. Rev. Microbiol. 18, 275-285. doi: 10.1038/ s41579-019-0288-0

Tiberi, S., du Plessis, N., Walzl, G., Vjecha, M. J., Rao, M., Ntoumi, F., et al. (2018a). Tuberculosis: progress and advances in development of new drugs, treatment regimens, and host-directed therapies. Lancet Infect. Dis. 18, e183-e198. doi: 10.1016/S1473-3099(18)30110-5

Tiberi, S., Muñoz-Torrico, M., Duarte, R., Dalcolmo, M., D’Ambrosio, L., and Migliori, G. -B. (2018b). New drugs and perspectives for new anti-tuberculosis regimens. Pulmonology 24, 86-98. doi: 10.1016/j.rppnen.2017.10.009

Torfs, E., Piller, T., Cos, P., and Cappoen, D. (2019). Opportunities for overcoming Mycobacterium tuberculosis drug resistance: emerging mycobacterial targets and host-directed therapy. Int. J. Mol. Sci. 20:2868. doi: $10.3390 /$ ijms 20122868

Tuyiringire, N., Tusubira, D., Munyampundu, J. -P., Tolo, C. U., Muvunyi, C. M., and Ogwang, P. E. (2018). Application of metabolomics to drug discovery and understanding the mechanisms of action of medicinal plants with anti-tuberculosis activity. Clin. Transl. Med. 7:29. doi: 10.1186/ s40169-018-0208-3

Uddin, R., Siddiqui, Q. N., Sufian, M., Azam, S. S., and Wadood, A. (2019). Proteome-wide subtractive approach to prioritize a hypothetical protein of XDR-Mycobacterium tuberculosis as potential drug target. Genes Genomics 41, 1281-1292. doi: 10.1007/s13258-019-00857-z

Vamathevan, J., Clark, D., Czodrowski, P., Dunham, I., Ferran, E., Lee, G., et al. (2019). Applications of machine learning in drug discovery and development. Nat. Rev. Drug Discov. 18, 463-477. doi: 10.1038/ s41573-019-0024-5

Van Der Meeren, O., Hatherill, M., Nduba, V., Wilkinson, R. J., Muyoyeta, M., Van Brakel, E., et al. (2018). Phase $2 b$ controlled trial of M72/AS01E vaccine to prevent tuberculosis. N. Engl. J. Med. 379, 1621-1634. doi: 10.1056/ NEJMoa1803484

van Opijnen, T., Bodi, K. L., and Camilli, A. (2009). Tn-seq: high-throughput parallel sequencing for fitness and genetic interaction studies in microorganisms. Nat. Methods 6, 767-772. doi: 10.1038/nmeth.1377

Viswanathan, G., Yadav, S., and Raghunand, T. R. (2017). Identification of mycobacterial genes involved in antibiotic sensitivity: implications for the treatment of tuberculosis with $\beta$-lactam-containing regimens. Antimicrob. Agents Chemother. 61, e00425-e00517. doi: 10.1128/AAC.00425-17

Viveiros, M., Leandro, C., and Amaral, L. (2003). Mycobacterial efflux pumps and chemotherapeutic implications. Int. J. Antimicrob. Agents 22, 274-278. doi: 10.1016/s0924-8579(03)00208-5

Vjecha, M. J., Tiberi, S., and Zumla, A. (2018). Accelerating the development of therapeutic strategies for drug-resistant tuberculosis. Nat. Rev. Drug Discov. 17, 607-608. doi: 10.1038/nrd.2018.28

World Health Organization (2019). Global tuberculosis report 2019.

Wu, Z., Shon, J. C., and Liu, K.-H. (2014). Mass spectrometry-based lipidomics and its application to biomedical research. J. Lifestyle Med. 4, 17-33. doi: 10.15280/jlm.2014.4.1.17

Yang, Y., Niehaus, K. E., Walker, T. M., Iqbal, Z., Walker, A. S., Wilson, D. J., et al. (2018). Machine learning for classifying tuberculosis drug-resistance from DNA sequencing data. Bioinformatics 34, 1666-1671. doi: 10.1093/ bioinformatics/btx801

Young, C., Walzl, G., and Du Plessis, N. (2020). Therapeutic host-directed strategies to improve outcome in tuberculosis. Mucosal Immunol. 13, 190-204. doi: 10.1038/s41385-019-0226-5

Zampieri, M., Szappanos, B., Buchieri, M. V., Trauner, A., Piazza, I., Picotti, P., et al. (2018). High-throughput metabolomic analysis predicts mode of action of uncharacterized antimicrobial compounds. Sci. Transl. Med. 10:eaal3973. doi: $10.1126 /$ scitranslmed.aal3973 
Zhang, W., Bai, Y., Wang, Y., and Xiao, W. (2016). Polypharmacology in drug discovery: a review from systems pharmacology perspective. Curr. Pharm. Des. 22, 3171-3181. doi: 10.2174/1381612822666160224142812

Zhu, X., Gerstein, M., and Snyder, M. (2007). Getting connected: analysis and principles of biological networks. Genes Dev. 21, 1010-1024. doi: 10.1101/ gad. 1528707

Zuniga, E. S., Early, J., and Parish, T. (2015). The future for early-stage tuberculosis drug discovery. Future Microbiol. 10, 217-229. doi: 10.2217/fmb.14.125

Zwerling, A., Behr, M. A., Verma, A., Brewer, T. F., Menzies, D., and Pai, M. (2011). The BCG world atlas: a database of global BCG vaccination policies and practices. PLoS Med. 8:e1001012. doi: 10.1371/journal.pmed.1001012
Conflict of Interest: The authors declare that the research was conducted in the absence of any commercial or financial relationships that could be construed as a potential conflict of interest.

Copyright (C) 2021 Minias, Żukowska, Lechowicz, Gąsior, Knast, Podlewska, Zygata and Dziadek. This is an open-access article distributed under the terms of the Creative Commons Attribution License (CC BY). The use, distribution or reproduction in other forums is permitted, provided the original author(s) and the copyright owner(s) are credited and that the original publication in this journal is cited, in accordance with accepted academic practice. No use, distribution or reproduction is permitted which does not comply with these terms. 